\title{
QUANTITATIVE ANALYSIS OF ETHNOBOTANY AND COMMON REMEDIES ASSOCIATED WITH THE THREATENED FLORA OF GUJRANWALA REGION, PUNJAB, PAKISTAN
}

\author{
IQBAL, M. S. ${ }^{*}$ - DAR, U. M. - AkBAR, M. - KhaliL, T. - ARShAD, N. - HuSSAIN, S. A. - \\ MUNIR, S. - ALI, M. A. \\ Department of Botany, University of Gujrat, Gujrat, Pakistan \\ (phone: +92-333-511-2154) \\ *Corresponding author \\ e-mail: drsajjad.iqbal@uog.edu.pk \\ (Received 12 ${ }^{\text {th }}$ Apr 2020; accepted 29 $9^{\text {th }}$ Jul 2020)
}

\begin{abstract}
Current studies revealed the ethnobotanical importance of the threatened flora of Gujranwala region, Punjab, Pakistan including Gujranwala, Kamoki and Wazirabad Townships. This region is facing rapid expansion of industrialization and urbanization, therefore it is prime time to document and conserve it before it is lost. 100 different species belonging to 52 families were recorded through questionnaire and interviews. Steps for preservation, classification, quantitative indices and phytochemical composition were performed. Asteraceae was the dominant family with ten species. 55\% of these represent herbs, $27 \%$ shrubs, $15 \%$ trees, $2 \%$ grasses and $1 \%$ weeds whereas $85 \%$ plant species were wild and $15 \%$ were cultivated. Leaves are the most frequently used parts $77 \%$, followed by stem $12 \%$, roots $12 \%$, flowers $20 \%$, rhizome $17 \%$, seed oil $18 \%$, and so on. As common remedies they are used as diuretics $26 \%$, against fever $25 \%$, laxatives $23 \%$, emollients $22 \%$, against constipation $20 \%$, blood purifiers $20 \%$, and against cough and cold $17 \%$ etc. RFC was recorded from 0.001 to 0.78 . Informant consensus factor (FCI) ranged from 10-40, with the lowest value belonging to Cиситиs melo which is used for treating eczema, dysuria, leucorrhea and as laxative whereas the highest 37 for Indigofera heterantha and Quercus incana reported to be used for hemorrhagic septicemia and joint pain. Concrete efforts are required to conserve traditional flora and to provide awareness for possible benefits of threatened species.
\end{abstract}

Keywords: ethnobotanical knowledge, documentation, urbanization, economic benefits, Gujranwala, Pakistan

\section{Introduction}

\section{Ethnobotany and drugs derived from plants}

The importance of plant based drugs is increasing day by day and their use spreads out of rural areas (Brandão et al., 2006; Shanley and Luz, 2003). These drugs are cheap, have minimum side effects and are usually more effective. Samie et al. (2005) reported that plant essential oils are used for treating sexually transmitted diseases, diarrhea, and dysentery like leaves of Acacia senegalensis. Both primary and secondary metabolites are major sources. Secondary metabolites are mostly used as phytomedicines that can be extracted from different plant parts including roots, stem, leaves, flowers, seeds and fruits etc. These plant based drugs include aspirin from willow bark, digoxin from foxglove, quinine from cinchona bark, and morphine from the opium poppy. Plants are natural source of remedies including cough, sneezing, head ache and even are successfully used against cancer. Plants are diverse in nature effective against more than one disease at a time (Khan et al., 2012). Pakistan has more than six to seven thousand species of plants that are wild in nature and about six hundred are known to have medicinally uses. Traditional knowledge is being transfer through Hakims who are Tabib (Traditional Medicine Practioner). Most of traditional knowledge is transferred through verbal 
communication and no comprehensive documentation is available to plants of particular area or this region and moreover comparison of topology, genetic variation for specific efficacy (Amiri and Joharchi, 2016). Ethnobotanical studies although have been carried out in different parts of Pakistan yet they are scarce and not properly documented.

It is known that plants of any type and species are suitable for medicines due to diverse biochemical composition. For example, an important genus of the family Moringaceae is Moringa and the species i.e. Moringa oleifera is an important medicinal plant that is native to the tropical areas and grows in all type of soils (Fahey, 2005). Moringa tree is regarded as one of the world's most reliable tree for medicines, as the whole plant can be utilized for food purposes or has some other advantageous characteristics (Gupta et al., 2010). Ethnobotanical account of 40 species relating to 26 families from Township Dargai, District Malakand Pakistan has been documented by Zaman et al. (2013). Likewise Wazir et al. (2007) recorded 20 medicinal salt tolerant plants found in the neighboring areas of District Karak. Akhtar and Begum (2009) reported that 55 species of plants relating to 38 families of plants were utilized for about 42 diseases in Jalala area District Mardan. Ahmed et al. (2013) identified 100 ethno medicinal plant species from Madyan valley in District Swat, Pakistan. Most of them were used as tonic, stimulant, narcotics, laxative and diuretic. Important knowledge about medicinal plants and their utilization from Dera Ghazi Khan, Punjab, Pakistan were documented by Gulshan et al. (2012). Zereen and Sardar (2013) enlisted the ethno botanical data of natives on wild trees in 08 Districts of Central Punjab that were Narowal, Sialkot, Sahiwal, Nankana Sahib, Faisalabad, Lahore, Pakpattan and Vehari. There were about 48 species of plants belonging to 23 families were gathered, including their utilization by people of particular districts for various activities i.e. fruits, vegetables, timber, medicine, fuel, fodder, etc. About 161 plant species from 57 different families involving 22 trees, 104 herbs, 23 shrubs, 3 parasitic and 9 grasses species from Township Takht-e-Nasrati, District Karak, and Pakistan were examined by Khan and Hussain (2013). The people of this region utilized 118 species (73.3\%) as traditional remedial plants, 114 species as animal food $(70.8 \%), 47$ species as fuel (26.7\%), 16 as timber (9.94\%), 23 as vegetables (14.3\%), 50 as veterinary medicines $(31.06 \%)$ and 90 species are recognized as honey bee attractive species (55.9\%).

\section{Uses of plant parts and selection of the area}

Seventy-one species of medicinal plants belonging to 38 families have been reported that were collected through different people. Mostly the favored part of plants used as traditional medicine were leaves $(38 \%)$ followed by the seed $(13 \%)$, whole plant $(11 \%)$, flower $(9 \%)$, fruit $(8 \%)$, root and bark (6\%). These were obtained through wild herbs $(54 \%)$ followed by the wild shrubs and wild trees (13\%), cultivated herbs (10\%), cultivated trees (5\%), cultivated shrubs (3\%) and wild grasses $(2 \%)$. The medicines or the herbal products are usually recommended by oral means to the patients (Mahmood et al., 2013).

Current studies focused on Gujranwala region that constitutes three Townships viz., Gujranwala, Kamoki and Wazirabad. Gujranwala is known as one of the most important industrial cities of Pakistan. Due to various anthropogenic activities especially urbanization, industrial waste and different types of pollution plants are losing their habitats and are at the verge of endangerment. So it is the need of the hour to cut short our all sources that cause pollution or to collect and preserve the plants that are being destroyed. For that purpose, ethnobotanical studies are the best approach for obtaining data, sample collection and preservation. This will ensure availability of enlisted species 
and documentation related to their significance, traditional uses, phytochemical composition and status of flora of different type of species/habitat within the District by both urban and rural people. This study aims to systematically document local flora of selected sites, traditional uses and their quantitative significance for illustrating importance of data collected for future application. This report would also be important to devise future strategy to plan survey, site selection, collection of sampling, interaction with local community and conservation efforts to ensure proper preservation for not only coming generations but also to earn revenue from herbal and pharmaceutical sector, along with comprehensive mode of action to resolve issues raised through anthropogenic activities.

\section{Materials and methods}

\section{Geological distribution and sites for survey}

Gujranwala region comprises of three Townships viz., Gujranwala, Kamonki and Wazirabad, which were selected for documenting traditional knowledge (Fig. 1). Gujranwala is an industrial city situated in the largest province of Punjab. Gujranwala is $226 \mathrm{~m}$ above sea level spreading over an area of $3198 \mathrm{~km}^{2}$ with a population of $1,960,136$. Gujranwala is Pakistan's $7^{\text {th }}$ largest populous and metropolitan area and the fifth largest city. The months May, June and July are the hottest months with the temperature ranging from $36-42{ }^{\circ} \mathrm{C}\left(97-108{ }^{\circ} \mathrm{F}\right)$ while it may drop below zero sometimes in winter. Therefore, climate of the district is usually hot and semiarid. Soil is mainly fertile due to its plain and simple topology that supports plant biodiversity. Across Gujranwala, mostly people utilize plants as medicine for therapeutic purposes residing both in rural and urban areas. Plants are generally used for food, paper, clothing, dying, timber, fodder for livestock, medicines, cosmetics, and other domestic purposes. Many species are linked to cultural heritages. Due to little information Gujranwala region is selected to document local flora with ethnobotanical significance. Native people including male, female, farmers and herbalists were contacted for interview and questionnaire completion.

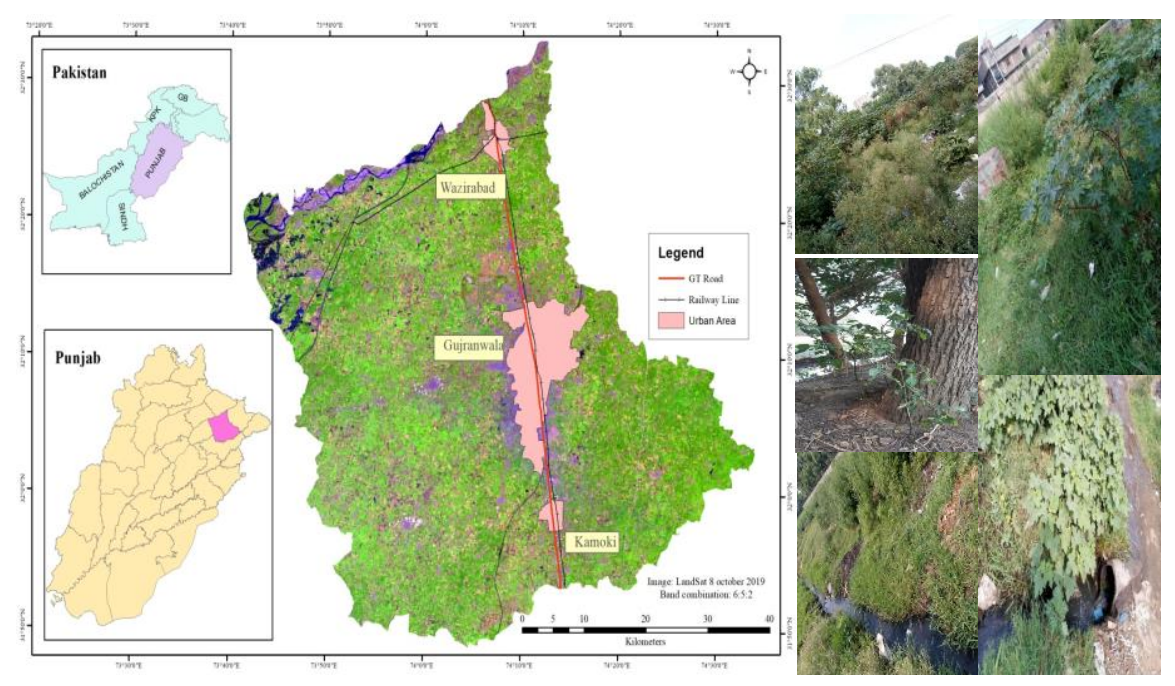

Figure 1. Map of Gujranwala region showing selected sites and habitats for survey (Gujranwala, Wazirabad, and Kamonki townships) 
The following materials were used in the survey: pencil, notebook, gloves, polythene bags and knife. The study of the selected sites were carried out from winter (November) 2018 to summer (July) 2019. Information regarding ethnobotanical uses were collected and noted in the form of questionnaires by the informants of the areas of different ages. For ethnobotanical knowledge herbalists, native people of old ages and hakims were also consulted. The method of classification was based upon the botanical rules and regulations.

\section{Collection, preservation and quantitative indices}

Collection was made during November 2018 to July 2019 through regular visits and field trips by selecting sites one by one. Plants were then dried under shade and converted to powder by grinding. Plants after their collection were washed by water, thoroughly cleaned and leaves were stripped by hands for better results. Questionnaire method was employed to note data for informants of the areas of all ages. For proper identification herbarium collections were consulted, many were matched with online databases and Flora of Pakistan. Herbarium specimen were prepared and deposited in Herbarium, Department of Botany, University of Gujrat, Gujrat, Pakistan. Herbarium specimen preparation was achieved by the following steps: (a) Voucher Number, (b) Common Names, (c) Botanical Names, (d) Habitats/Locality, (e) Family Names, and (f) The region etc. Quantitative indices were performed by employing various formulae as presented below.

\section{Informant consensus factor (FCI/ICF)}

FCI values were calculated as the following formula (Heinrich et al., 2009).

$$
\mathrm{FCI}=\mathrm{N}_{\mathrm{ur}}-\mathrm{N}_{\mathrm{t}} / \mathrm{N}_{\mathrm{ur}}-1
$$

where, $\mathrm{N}_{\mathrm{ur}}$ is the total number of use reports for each disease category. $\mathrm{N}_{\mathrm{t}}$ is the number of species used in the said category.

\section{Relative frequency of citation (RFC)}

The local importance of the species was calculated with the help of the relative frequency of citation. To find the RFC, number of responder's had provided useful information about species (FC) was divided by the total number of responder's in the field survey $(\mathrm{N})$ as calculated previously (Tardío et al., 2008).

$$
\mathrm{RFC}=\mathrm{FC} / \mathrm{N}
$$

\section{Relative importance level (RIL)}

To find the RIL, number of responder's had provided useful information about species $(\mathrm{FC})$ was divided by the total number of responders of all species $\left(\mathrm{FC}_{\mathrm{t}}\right)$ as described by (Friedman, 1986).

$$
\mathrm{RIL}=\mathrm{FC} / \mathrm{FC}_{\mathrm{tn}}
$$




\section{Use value $(U V)$}

Use value can be calculated by:

$$
\mathrm{UV}=\Sigma \mathrm{U}_{\mathrm{i}} / \mathrm{n}_{\mathrm{i}}
$$

$\Sigma U_{\mathrm{i}}$ is the use value of the species. $\mathrm{n}_{\mathrm{i}}$ is the number of responders.

Fidelity level (FL)

It is the ratio of the respondents showing the uses of a specific plant to treat a particular disease. FL is calculated by following Alexiades (1996).

$$
\mathrm{FC}(\%)=\mathrm{FC}_{\mathrm{P}} / \mathrm{FC} \times 100
$$

where $\mathrm{FC}_{\mathrm{p}}$ is the frequency of citation of particular disease. $\mathrm{FC}$ is the total frequency of citation for a particular disease.

\section{Corrected fidelity level (CFL)}

It is used for a correction factor to rank the plant with different RIL and FL values. It can be calculated by Ali-Shtayeh (2000).

$$
\mathrm{CFL}=\mathrm{FL} \times \mathrm{RIL}
$$

Use reports (UR)

It is the total number of uses reported by the user.

At room temperature, the plants were dried and grinded in to powder form. Then $200 \mathrm{~g}$ of each powdered plant material was soaked in $200 \mathrm{ml}$ ethanol for $24 \mathrm{~h}$. Then this was filtered using a filter paper i.e. Whatmann's filter paper No.42. This plant extract and the filtrate was then placed in vacuum at about $30^{\circ} \mathrm{C}$ and stored at $4{ }^{\circ} \mathrm{C}$. The following procedures were applied for preliminary screening of phytochemicals:

\section{Tests for alkaloids}

$0.2 \mathrm{~g}$ powdered sample of each plant was mixed with $10 \mathrm{ml}$ of $1 \% \mathrm{HCl}$. Then this sample was transferred to water bath for a few minutes and $1 \mathrm{ml}$ of this extract was treated with 2-4 drops of Dragendroff's reagent. The orange reddish precipitates confirmed the presence of alkaloids (Aiyegoro and Okoh, 2010).

\section{Tests for flavonoids}

For flavonoids presence two solutions were made. Solution A was prepared by the help of $5 \mathrm{ml}$ ethanol extract that was prepared earlier. Solution B contained $5 \mathrm{ml}$ of ethanol solvent and $5 \mathrm{ml}$ of $\mathrm{KOH}$. Both these solutions were mixed together and the presence of yellow color indicated the flavonoids (Jaffer et al., 1983).

\section{Tests for tannins}

$10 \mathrm{ml}$ of the solution in extract form was taken and about 2 drops of ferric chloride solution was put into the extract. The blue colored solution detected the presence of tannins (Aiyegoro and Okoh, 2010). 


\section{Tests for terpenoids}

$1 \mathrm{ml}$ of acid anhydride plus concentrated sulphuric acid was treated with $1 \mathrm{ml}$ of the extract. Reddish Brown color appeared which confirmed the presence of terpenoids (Aiyegoro and Okoh, 2010).

\section{Tests for saponins}

The extract was added in $2 \mathrm{ml}$ distilled water shaken well. Foam was the indication that the sample contains saponins in it (Shihata, 1951).

\section{Tests for phenols}

$5 \mathrm{ml}$ of the extracts of the plants were taken in a test tube and $1 \mathrm{ml}$ of $1 \%$ solution of ferric chloride $1 \%$ solution of potassium ferrocyanide was added. Reddish blue color indicated the presence of phenols in it (Farhan et al., 2012).

\section{Tests for resins}

$20 \mathrm{ml}$ of HCL was added in the $10 \mathrm{ml}$ extract of each plant. Turbidity indicated the presence of resins in the sample (Alsaidy, 2013).

\section{Tests for steroids}

$2 \mathrm{ml}$ of acetic anhydride plus $2 \mathrm{ml}$ of sulphuric acid was added in the $0.5 \mathrm{~g}$ aqueous extracts of the plants. The change in the color from violet to blue or green indicated the presence of steroids (Sofowara, 1993).

\section{Results}

\section{Ethnobotanical elaboration and therapeutic uses}

Questionnaire was filled by different age group of people with varying types of qualification, educational background, gender, informant category (Table 1). Data were collected from natives of the selected sites i.e. Gujranwala, Kamonki and Wazairabad Townships through surveys. Total natives that provide useful information in this survey were 300, out of which 185 were females $(61.67 \%)$ and 115 were males $(38.33 \%)$. Traditional health practitioners included 45 persons and 245 were indigenous people in which people below 20 years of age were 30, people between 21 and 30 years old were 27 , people in the category of 31 to 40 years were 73 , people of age between 41 and 50 were 70, people of age between 51 and 60 were 66 and above 61 were 34. Regarding their educational background 165 illiterate persons were included, people of matriculation level were 70, 30 people were of intermediate level, 20 people were of bachelors level and 15 people were of masters level. Therefore, educational background also reflects understanding towards importance and application of natural resources among different age groups.

Collected plant species can be divided into different life forms/types viz., herbs $(55 \%)$, shrubs $(27 \%)$, trees $(15 \%)$, grasses $(2 \%)$ and weeds $(1 \%)$. Wild and cultivated plants are also found in the study area which is $85 \%$ and $15 \%$ respectively (Fig. 2). Different plant parts such as roots, shoots, stem, leaves and flowers were used for various preparations. Leaves were the most frequently used parts $(77 \%)$ followed by 


$$
\text { - } 7959 \text { - }
$$

stem (12\%), roots (12\%), flower (20\%), rhizome (17\%), seed oil (18\%), shoots (27\%), gel (8\%) and whole plant (24\%) (Fig. 3). These parts were found to be used in the form of different preparations such as extract (67\%), juice (40\%), powder (58\%), poultices (30\%), decoction (57\%), ash (25\%), mixture with sugar (3\%), infusion (48\%) (Fig. 4).

Family Asteraceae contributes the highest number of medicinal plant species that included 10 species, followed by Malvaceae with 6, Solanaceae 5, Mimosaceae, Cucurbitaceae with 4, Poaceae, Euphorbiaceae and Amaranthaceae with 3 each, Fabaceae, Rosaceae, Tiliaceae, and Portulaceae with 2 each and Asparagaceae, Brassicaceae, Rutaceae have 1 species each and so on. The species found were whether monocot or dicot at particular location are shown in Table 2.

Table 1. Demographic data of the respondents from the study area

\begin{tabular}{|c|c|c|c|c|}
\hline S. No. & Variable & Categories & Number of persons & $\%$ age \\
\hline \multirow{2}{*}{1.} & \multirow{2}{*}{ Gender } & Male & 185 & 61.67 \\
\hline & & Female & 115 & 38.33 \\
\hline \multirow{2}{*}{2.} & \multirow{2}{*}{ Informant category } & Traditional health practitioners & 45 & 15 \\
\hline & & Indigenous people & 255 & 85 \\
\hline \multirow{6}{*}{3.} & \multirow{6}{*}{ Age } & $\leq 20$ years & 30 & 10 \\
\hline & & $21-30$ years & 27 & 9 \\
\hline & & $31-40$ years & 73 & 24.33 \\
\hline & & $41-50$ years & 70 & 23.33 \\
\hline & & $51-60$ years & 66 & 22 \\
\hline & & $\geq 61$ years & 34 & 11.33 \\
\hline \multirow{5}{*}{4.} & \multirow{5}{*}{ Educational background } & Illiterate & 165 & 55 \\
\hline & & Matric & 70 & 23.33 \\
\hline & & Intermediate & 30 & 10 \\
\hline & & Bachelors & 20 & 6.66 \\
\hline & & Masters & 15 & 5 \\
\hline
\end{tabular}

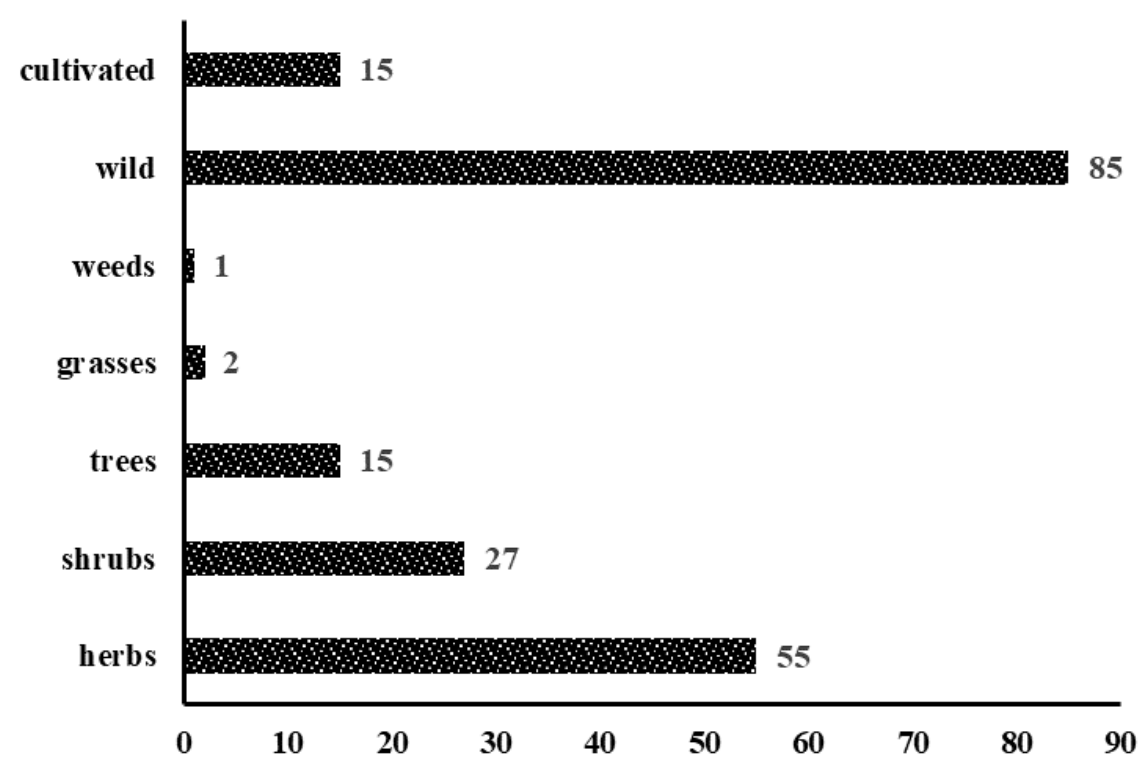

Figure 2. Different plant types studied in 3 selected sites of Gujranwala region 


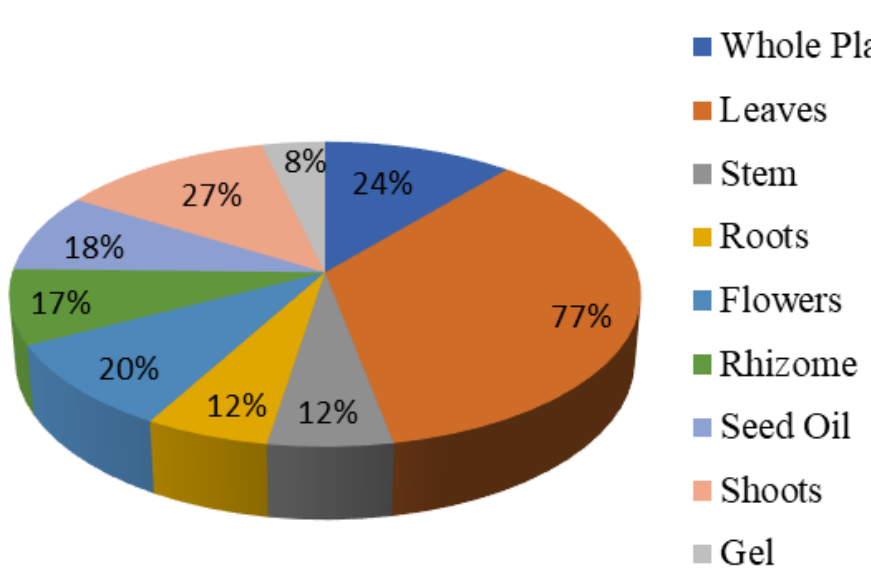

Figure 3. Plant parts used for different therapeutic purposes studied in 3 selected sites of Gujranwala region

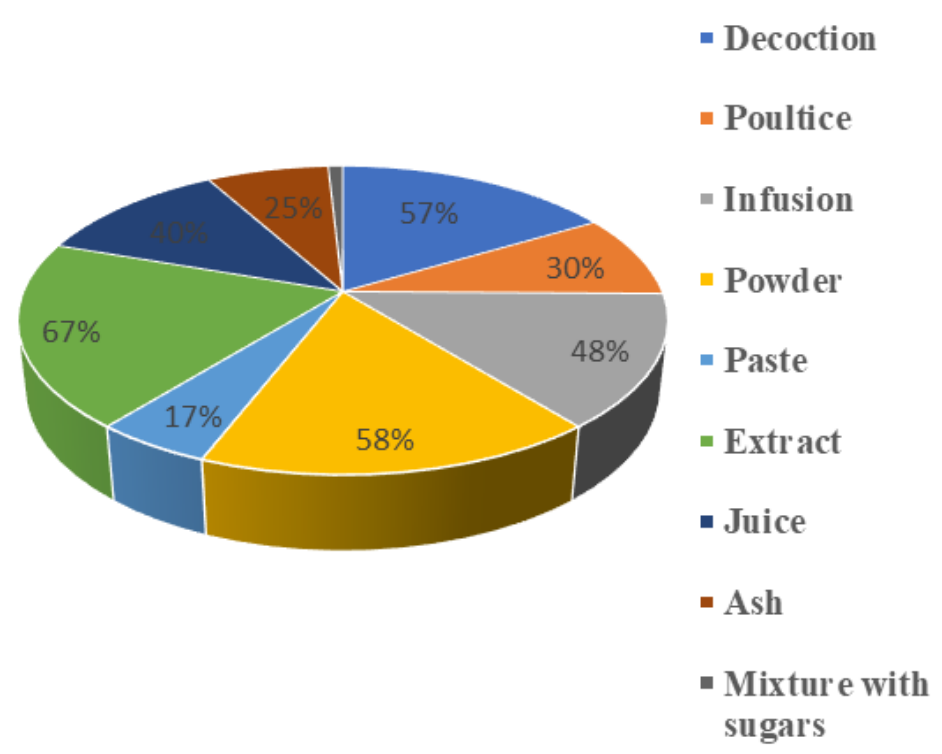

Figure 4. Graphical presentation of plant preparations used for different therapeutic purposes from 3 selected sites of Gujranwala region

Table 2. Families count present in the selected sites of Gujranwala

\begin{tabular}{|c|c|c|c|c|c|c|c|}
\hline S.No. & Family & Genus & Species & Dicot/Monocot & Wazirabad & Gujranwala & Kamonki \\
\hline 1. & \multirow{10}{*}{ Astereace } & Xanthium & strumarium & Eudicot & + & + & + \\
\hline 2. & & Parthenium & hysterophorus & Eudicot & + & + & + \\
\hline 3. & & Artemisia & scoparia & Eudicot & + & - & + \\
\hline 4. & & Eclipta & prostrate & Dicot & + & - & + \\
\hline 5. & & Carthamus & oxyacantha & Dicot & + & + & - \\
\hline 6. & & Taraxacum & officinale & Dicot & + & + & - \\
\hline 7. & & Calendula & arvensis & Dicot & - & + & + \\
\hline 8. & & Conyza & aegyptiaca & Dicot & + & + & + \\
\hline 9. & & Bombax & ceiba & Dicot & + & + & + \\
\hline 10. & & Cichorium & intybus & Dicot & + & - & + \\
\hline
\end{tabular}




\begin{tabular}{|c|c|c|c|c|c|c|c|}
\hline 11. & \multirow{6}{*}{ Malvaceae } & Hibiscus & rosasinensis & Dicot & + & + & + \\
\hline 12. & & Abutilon & indicum & Dicot & + & + & - \\
\hline 13. & & Marva & parviflora & Dicot & + & + & + \\
\hline 14. & & Melia & azedarach & Dicot & + & + & + \\
\hline 15. & & Althea & rosea & Dicot & + & + & + \\
\hline 16. & & Malvastrum & coromandelianum & Dicot & - & + & + \\
\hline 17. & \multirow{5}{*}{ Solanaceae } & Withania & somnifera & Dicot & + & - & + \\
\hline 18. & & Solanum & surattense & Dicot & + & + & - \\
\hline 19. & & Solanum & nigrum & Dicot & + & + & + \\
\hline 20. & & Capsicum & frutescens & Dicot & + & + & + \\
\hline 21. & & Datura & innoxia & Dicot & + & + & + \\
\hline 22. & \multirow{5}{*}{ Papilionaceae } & Indigofera & linifolia & Dicot & - & - & + \\
\hline 23. & & Dalbergia & sissoo & Dicot & + & + & + \\
\hline 24. & & Pongamia & pinnata & Dicot & + & - & + \\
\hline 25. & & Crotolaria & burhia & Dicot & + & - & + \\
\hline 26. & & Alhagi & maurorum & Monocot & + & - & - \\
\hline 27. & \multirow{4}{*}{ Cucurbitceae } & Citrullus & colocynthis & Dicot & + & + & + \\
\hline 28. & & Cucurbita & Реро & Dicot & + & + & + \\
\hline 29. & & Cucumus & Melo & Dicot & + & + & + \\
\hline 30. & & Benincasa & hispida & Dicot & - & + & + \\
\hline 31. & \multirow{4}{*}{ Mimosaceae } & Albizia & Lebbek & Dicot & + & + & + \\
\hline 32. & & Cassia & fistula & Dicot & + & + & + \\
\hline 33. & & Acacia & modesta & Dicot & + & + & + \\
\hline 34. & & Acacia & nilotica & Dicot & + & + & + \\
\hline 35. & \multirow{3}{*}{ Poaceae } & Cynodon & dactylon & Monocot & + & + & + \\
\hline 36. & & Avena & sativa & Monocot & + & - & + \\
\hline 37. & & Bumbusa & arundinacea & Monocot & - & + & + \\
\hline 38. & \multirow{3}{*}{ Euphorbiaceae } & Euphorbia & hirta & Dicot & + & + & + \\
\hline 39. & & Euphorbia & heliscopia & Dicot & + & + & + \\
\hline 40. & & Ricinus & communis & Dicot & + & + & - \\
\hline 41. & \multirow{3}{*}{ Amaranthaceae } & Amaranthus & viridis & Dicot & + & + & + \\
\hline 42. & & Achyranthes & aspera & Dicot & + & + & + \\
\hline 43. & & Scandix & pectenveneris & Dicot & - & - & + \\
\hline 44. & \multirow{2}{*}{ Fabaceae } & Melilotus & indica & Dicot & - & + & + \\
\hline 45. & & Indigofera & heterantha & Dicot & + & - & + \\
\hline 46. & \multirow{2}{*}{ Rosaceae } & Duchesnea & indica & Dicot & + & + & - \\
\hline 47. & & Rosa & indica & Dicot & + & + & + \\
\hline 48. & \multirow{2}{*}{ Tiliaceae } & Grewia & asiatica & Monocot & - & + & + \\
\hline 49. & & Corchorus & aestuans & Dicot & + & + & + \\
\hline 50. & \multirow{2}{*}{ Lamiaceae } & Micromeria & biflora & Dicot & - & + & - \\
\hline 51. & & Mentha & spicata & Dicot & + & + & + \\
\hline 52. & \multirow{2}{*}{ Papaveraceae } & Fumaria & indica & Dicot & + & + & + \\
\hline 53. & & Argemone & mexicana & Dicot & + & - & + \\
\hline 54. & \multirow{3}{*}{ Apocynaceae } & Vinca & major & Dicot & + & + & + \\
\hline 55. & & Nerium & oleander & Dicot & + & + & + \\
\hline 56. & & Rhazya & stricta & Monocot & + & - & + \\
\hline 57. & \multirow{2}{*}{ Amaryllidaceae } & Allium & cepa & Monocot & + & + & + \\
\hline 58. & & Allium & sativum & Monocot & + & + & + \\
\hline 59. & \multirow{2}{*}{ Asclepiadaceae } & Calotropis & procera & Eudicot & + & - & + \\
\hline 60. & & Caralluma & edulis & Eudicot & + & + & - \\
\hline 61. & \multirow{2}{*}{ Moraceae } & Ficus & benghalensis & Dicot & + & + & + \\
\hline 62. & & Morus. & nigra & Dicot & + & + & + \\
\hline
\end{tabular}




\begin{tabular}{|c|c|c|c|c|c|c|c|}
\hline 63. & \multirow{2}{*}{ Myrtaceae } & Syzygium & cumini & Dicot & + & - & + \\
\hline 64. & & Eucalyptus & globulus & Dicot & & + & + \\
\hline 65. & \multirow{2}{*}{ Portulacaceae } & Portulaca & oleracea & Dicot & + & + & - \\
\hline 66. & & Portulaca & quadrifida & Dicot & + & + & - \\
\hline 67. & \multirow{2}{*}{ Boraginaceae } & Trichodesma & indicum & Dicot & - & + & + \\
\hline 68. & & Heliotropium & strigosum & Dicot & + & + & + \\
\hline 69. & Cuscutaceae & Cuscuta & reflexa & Dicot & + & + & + \\
\hline 70. & Cannabinaceae & Cannabis & sativa & Dicot & + & + & + \\
\hline 71. & Crassulaceae & Bryophyllum & pinnatum & Dicot & + & + & - \\
\hline 72. & Convolvulaceae & Convolvulus & album & Dicot & + & + & + \\
\hline 73. & Chenopodiaceae & Chenopodium & album & Dicot & + & + & + \\
\hline 74. & Cyperaceae & Cyperus & rotundus & Monocot & - & + & + \\
\hline 75. & Punicaceae & Punica & granatum & Dicot & + & + & + \\
\hline 76. & Nyctaginaceae & Mirabilis & jalapa & Dicot & + & - & - \\
\hline 77. & Rhamnaceae & Zizyphus & nummularia & Dicot & + & - & + \\
\hline 78. & Oxalidaceae & Oxalis & corniculata & Dicot & - & + & + \\
\hline 79. & Apiaceae & Foeniculum & vulgare & Dicot & + & + & + \\
\hline 80. & Aizoaceae & Trianthema & portulacastrum & Dicot & - & - & + \\
\hline 81. & Acanthaceae & Justicia & adhatoda & Dicot & + & + & - \\
\hline 82. & Salvadoraceae & Salvadora & oleoides & Dicot & + & + & + \\
\hline 83. & Tamaricaceae & Tamarix & dioica & Dicot & + & - & + \\
\hline 84. & Zygophyllaceae & Tribulus & camaldulensis & Dicot & + & + & - \\
\hline 85. & Arecaceae & Phoenix & dactylifera & Monocot & + & - & + \\
\hline 86. & Meliaceae & Azadirachta & indica & Monocot & + & + & + \\
\hline 87. & Asphodelaceae & Aloe & vera & Monocot & + & + & + \\
\hline 88. & Pontederiaceae & Eichhornia & crassipes & Monocot & - & + & + \\
\hline 89. & Asparagaceae & Asparagus & racemosus & Monocot & + & + & + \\
\hline 90. & Polygonaceae & Polygonum & plebium & Dicot & + & - & + \\
\hline 91. & Urticaceae & Debregeasia & salicifolia & Dicot & + & + & - \\
\hline 92 & Brassicaceae & Lepidium & didymium & Dicot & + & + & + \\
\hline 93 & Sapindaceae & Dodonaea & viscosa & Dicot & - & + & + \\
\hline 94. & Hypericaceae & Hypericum & oblongifolium & Dicot & + & - & + \\
\hline 95. & Oleaceae & Jasminum & officinale & Dicot & + & + & - \\
\hline 96. & Rananculaceae & Ranunculus & muricatus & Dicot & - & - & + \\
\hline 97. & Caprifoliaceae & Lonicera & quinquelocularis & Dicot & + & + & - \\
\hline 98. & Fagaceae & Quercus & incana & Dicot & + & - & + \\
\hline 99. & Berberidaceae & Berberis & lyceum & Dicot & + & + & - \\
\hline 100. & Rutaceae & Zanthoxylum & armatum & Dicot & - & + & + \\
\hline
\end{tabular}

Medicinal values of 100 species of plants belonging to different families were recorded, that was used for different purposes such as stimulant, anthelmintic, cardiovascular diseases, febrifuge, diuretic, vermifuge, sedative and even antidote. Many diseases were treated such as hemorrhagic septicemia, jaundice, wound healing, severe burns and skin treatments of various types. Important medicinal uses of the plants include cold, flu, fever, dysentery, male diseases like gonorrhea, sores on genitals and female diseases such as amenorrhea, leucorrhea, and diarrhea, tooth aches, diabetes etc. Plants associated with different diseases for their treatment found in the study area were classified as diuretic (26\%), febrifuge (25\%), laxative (23\%), emollient $(22 \%)$, constipation (20\%), blood purifier (20\%), tonics (19\%), diarrhea curing (19\%), 
carminatives $(18 \%)$, cough and cold curing medicines (17\%), dysentery treating (15\%), jaundice pain relievers (15\%), and anti-diabetics (14\%) (Table 3; Fig. 5).

Twenty different plants were shortlisted for phytochemical analysis based on usefulness against common diseases through questionnaire method. Phytochemicals especially secondary metabolites are of supreme importance against number of ailments. The detection for presence/absence of these compounds is provided as a graphical presentation in Figure 6.

Table 3. Scientific names, parts used, preparation used as therapeutic uses and their habit/habitat studied in the selected sites of Gujranwala

\begin{tabular}{|c|c|c|c|c|c|}
\hline S. No. & Scientific name & English names & Parts used & Preparation & Habit/habitat \\
\hline 1. & Convolvulus album & Field bindweed & $\begin{array}{c}\text { Whole Plant, Mostly } \\
\text { Leaves }\end{array}$ & Powder or Juice & $\begin{array}{c}\text { Shrub, } \\
\text { Wild/Cultivated }\end{array}$ \\
\hline 2. & Bryophyllum pinnatum & $\begin{array}{l}\text { Air plant/Cathedral } \\
\text { bells }\end{array}$ & Leaves & Extract, Paste & Herb, Cultivated \\
\hline 3. & Cannabis sativa & Cannabis & $\begin{array}{c}\text { Shoot, Leaves, } \\
\text { Seeds } \\
\end{array}$ & $\begin{array}{c}\text { Powder, Juice, } \\
\text { Decoction }\end{array}$ & Herb, Wild \\
\hline 4. & Chenopodium album & $\begin{array}{c}\text { Wild spinach/manure } \\
\text { weed }\end{array}$ & Whole Plant & $\begin{array}{c}\text { Powder, Cooked, } \\
\text { Extract }\end{array}$ & Herb, Wild \\
\hline 5. & Heliotropium strigosum & Bristly Heliotrope & Whole Plant, Leaves & Powder, Extract & Herb, Wild \\
\hline 6. & Xanthium strumarium & Rough cocklebur & Leaves, Seeds, Fruit & Paste, Extract & Herb, Wild \\
\hline 7. & Trichodesma indicum & Indian borage & Leaves & $\begin{array}{l}\text { Paste, Extract, } \\
\text { Decoction }\end{array}$ & Herb, Wild \\
\hline 8. & Citrullus colocynthis & $\begin{array}{l}\text { Bitter cucumber/wild } \\
\text { gourd }\end{array}$ & Seed, Fruit, Seed oil & Paste, Extract, Oil & Herb, Wild \\
\hline 9. & Benincasa hispida & Wax gourd/ash gourd & Fruit, Seed oil & Oil, Juice & Herb, Cultivated \\
\hline 10. & Cucumus melo & Muskmelon & Fruit, Leaves & Decoction, Paste & Herb, Wild \\
\hline 11. & Cucurbita pepo & Winter squash/pumpkin & Fruit, Leaves, Seeds & Paste & $\begin{array}{c}\text { Herb, } \\
\text { Wild/Cultivated }\end{array}$ \\
\hline 12. & Cuscuta reflexa & Giant dodder & Whole Plant & Powder, Extract & $\begin{array}{c}\text { Herb, } \\
\text { Wild/Cultivated }\end{array}$ \\
\hline 13. & Euphorbia hirta & Asthma plant & Leaves, Milky Latex & Extract & Herb, Wild \\
\hline 14. & Euphorbia heliscopia & Sun spurge & Seeds, Milky latex & Roasted, Extract & Herb, Wild \\
\hline 15. & Ricinus communis & Castor bean & Seeds, Stem, Leaves & Powder, Oil & Shrub, Wild \\
\hline 16. & Cyperus rotundus & Nut sedge & $\begin{array}{c}\text { Whole Plant, } \\
\text { Leaves, Rhizome }\end{array}$ & Decoction, Paste & Herb, Wild \\
\hline 17. & Indigofera linifolia & Indigo & Whole Plant, Leaves & Decoction, Extract & Herb, Wild \\
\hline 18. & Dalbergia sissoo & North Indian rosewood & Bark, Leaves & $\begin{array}{l}\text { Decoction, Juice, } \\
\text { Infusion, Powder }\end{array}$ & Tree, Wild \\
\hline 19. & Pongamia pinnata & $\begin{array}{c}\text { Indian beech/Pongam } \\
\text { oiltree }\end{array}$ & $\begin{array}{c}\text { Young Branches, } \\
\text { Leaves, Seeds, Bark }\end{array}$ & Decoction, oil, Powder & Tree, Wild \\
\hline 20. & Crotalaria burhia & Ethiopian rattlebox & $\begin{array}{c}\text { Flowers, Leaves, } \\
\text { Whole Plant }\end{array}$ & Extract, Juice & Herb, Wild \\
\hline 21. & Alhagi maurorum & Camelthorn bush & Flowers, Leaves & Decoction, Powder & Herb, Wild \\
\hline 22. & Argemone Mexicana & Mexican poppy & $\begin{array}{c}\text { Flowers, Milky } \\
\text { Latex }\end{array}$ & Powder, Extract & Herb, Wild \\
\hline 23. & Cynodon dactylon & Bermuda grass/Dhoob & $\begin{array}{c}\text { Roots, Flowers, } \\
\text { Leaves }\end{array}$ & Powder, Juice & Grass, Wild \\
\hline 24. & Avena sativa & Common oat & Leaves, Whole Plant & Powder, Infusion & Grass, Wild \\
\hline 25. & Bumbusa arundinacea & Bamboo & $\begin{array}{l}\text { Leaves, Young } \\
\text { Shoots, Roots }\end{array}$ & Powder, Infusion, Ash & Shrub, Wild \\
\hline 26. & Rosa indica & Rose & $\begin{array}{c}\text { Leaves, Flower, } \\
\text { Roots }\end{array}$ & $\begin{array}{l}\text { Powder, Extract, } \\
\text { Mixture with sugar }\end{array}$ & $\begin{array}{c}\text { Shrub, } \\
\text { Wild/Cultivated }\end{array}$ \\
\hline 27. & Zizyphus nummularia & Wild jujube & Leaves, Fruit & Decoction, Paste & Shrub, Wild \\
\hline 28. & Punica granatum & Pomegranate & Bark, Fruit & Powder, Ash & Tree, Cultivated \\
\hline
\end{tabular}


Iqbal et al.: Ethnobotany and common remedies associated with threatened flora of Gujranwala region, Punjab, Pakistan, elaborated through quantitative indices - 7964 -

\begin{tabular}{|c|c|c|c|c|c|}
\hline 29. & Portulaca oleracea & Common purslane & Whole plant, Leaves & Powder, Infusion & Herb, Wild \\
\hline 30. & Portulaca quadrifida & $\begin{array}{c}\text { Duckweed/little } \\
\text { hogweed }\end{array}$ & Seeds, Leaves & $\begin{array}{c}\text { Powder, Infusion, } \\
\text { Paste }\end{array}$ & Herb, Wild \\
\hline 31. & Eichhornia crassipes & $\begin{array}{c}\text { Common water } \\
\text { hyacinth }\end{array}$ & Leaves & Infusion, Paste & Herb, Wild \\
\hline 32. & Polygonum plebium & Common knotweed & Leaves, Whole Plant & $\begin{array}{c}\text { Extract, Oil, } \\
\text { Decoction, Ash }\end{array}$ & Herb, Wild \\
\hline 33. & Hibiscus rosasinensis & $\begin{array}{c}\text { Shoe flower/Chinese } \\
\text { hibiscus }\end{array}$ & Fruit & Extract, Juice & $\begin{array}{c}\text { Shrub, } \\
\text { Wild/Cultivated }\end{array}$ \\
\hline 34. & Abutilon indicum & Mallow & Seeds, Leaves & Decoction & Herb, Wild \\
\hline 35. & Marva parviflora & $\begin{array}{c}\text { Cheeseweed/marshmall } \\
\text { ow }\end{array}$ & Leaves & Decoction, Extract & Herb, Wild \\
\hline 36. & Melia azedarach & Chinaberry tree & Leaves & Paste, Infusion & Tree, Wild \\
\hline 37. & Mentha spicata & Bush mint/spearmint & Leaves, Shoot & Decoction, Paste & $\begin{array}{c}\text { Herb, } \\
\text { Wild/Cultivated }\end{array}$ \\
\hline 38. & Mirabilis jalapa & $\begin{array}{c}\text { Marvel of peru/four } \\
\text { o'clock flower }\end{array}$ & Leaves, Flower & Decoction, Paste, Juice & $\begin{array}{c}\text { Herb, } \\
\text { Wild/Cultivated }\end{array}$ \\
\hline 39. & Syzygium cumini & Black plum/Java plum & Seeds & Powder & Tree, Cultivated \\
\hline 40. & Eucalyptus globulus & Southern blue gum & Leaves & $\begin{array}{c}\text { Decoction, Extract, } \\
\text { Fumes } \\
\end{array}$ & Tree, Cultivated \\
\hline 41. & Acacia nilotica & Gum Arabic tree & Flowers, Bark, Pod & $\begin{array}{l}\text { Decoction, Powder, } \\
\text { Juice }\end{array}$ & Tree, Wild \\
\hline 42. & Albizia lebbeck & Lebbek tree/flea tree & $\begin{array}{c}\text { Flowers, Seeds, } \\
\text { Bark } \\
\end{array}$ & Decoction & Tree, Wild \\
\hline 43. & Cassia fistula & $\begin{array}{c}\text { Indian laburnum/golden } \\
\text { shower }\end{array}$ & $\begin{array}{c}\text { Flowers, Seeds, } \\
\text { Fruits }\end{array}$ & Powder & Tree, Wild \\
\hline 44. & Acacia modesta & Phulai & Bark, Stem, Gum & Powder & Tree, Wild \\
\hline 45. & Ficus benghalensis & Banyan & $\begin{array}{c}\text { Milky Latex, Young } \\
\text { Branches }\end{array}$ & Powder, Gum & Tree, Wild \\
\hline 46. & Morus nigra & Black mulberry & Leaves, Roots, Fruit & $\begin{array}{c}\text { Juice, Infusion, } \\
\text { Powder }\end{array}$ & Tree, Wild \\
\hline 47. & Oxalis corniculata & Creeping woodsorrel & Leaves, Whole Plant & Extract, Powder & Herb, Wild \\
\hline 48. & Foeniculum vulgare & Sweat fennel & Leaves, Seeds & Extract, Decoction & Herb, Cultivated \\
\hline 49. & Trianthema portulacastrum & Desert horsepurslane & $\begin{array}{l}\text { Whole Plant, } \\
\text { Leaves, Roots }\end{array}$ & $\begin{array}{c}\text { Powder, Decoction, } \\
\text { Extract }\end{array}$ & Herb, Wild \\
\hline 50. & Justicia adhatoda & Malabar nut/adhatoda & $\begin{array}{c}\text { Flower, Leaves, } \\
\text { Roots }\end{array}$ & Juice, Decoction & Herb, Wild \\
\hline 51. & Allium sativum & Garlic & Bulbs & Juice & Herb, Wild \\
\hline 52. & Nerium oleander & Oleander & Roots, Stem, Leaves & Powder, Extract & Shrub, Wild \\
\hline 53. & Amaranthus viridis & Slender amaranth & Leaves & Powder, Paste & Herb, Wild \\
\hline 54. & Achyranthes aspera & Chaff flower & $\begin{array}{l}\text { Leaves, Roots, } \\
\text { Seeds }\end{array}$ & $\begin{array}{l}\text { Powder, Extract, } \\
\text { Decoction }\end{array}$ & Herb, Wild \\
\hline 55. & Calotropis procera & $\begin{array}{c}\text { Apple of } \\
\text { Sodom/roostertree }\end{array}$ & \begin{tabular}{|c|} 
Stem, Milky Latex, \\
Branches, Leaves, \\
Flowers \\
\end{tabular} & Poultices, Ash, Powder & Herb, Wild \\
\hline 56. & Caralluma edulis & Caralluma & Whole plant, Leaves & Juice, Extract, Powder & Shrub, Wild \\
\hline 57. & Parthenium hysterophorus & $\begin{array}{c}\text { Santa Maria } \\
\text { feverfew/whitetop } \\
\text { weed }\end{array}$ & Flowers, Leaves & Extract, Powder & Herb, Wild \\
\hline 58. & Artemisia scoparia & Virgate wormwood & \begin{tabular}{|c|} 
Flowers, Leaves, \\
Whole Plant, Shoot \\
\end{tabular} & $\begin{array}{c}\text { Extract, Powder, } \\
\text { Decoction }\end{array}$ & Herb, Wild \\
\hline 59. & Eclipta prostrate & False daisy & Roots, Whole Plant & Extract, Juice, Oil & Herb, Wild \\
\hline 60. & Carthamus oxyacantha & Wild safflower & Seeds & Oil & Herb, Wild \\
\hline 61. & Taraxacum officinale & Common dandelion & Leaves, Roots & $\begin{array}{c}\text { Paste, Powder, } \\
\text { Decoction }\end{array}$ & Herb, Wild \\
\hline 62. & Salvadora oleoides & Salvadora & Branches, Fruits & Decoction & Shurb, Wild \\
\hline 63. & Datura innoxia & Pricklyburr & Leaves, Seeds & Extract & Herb, Wild \\
\hline 64. & Capsicum frutescens & Chili pepper & Fruits & Powder, Paste & Herb, Cultivated \\
\hline
\end{tabular}


Iqbal et al.: Ethnobotany and common remedies associated with threatened flora of Gujranwala region, Punjab, Pakistan, elaborated through quantitative indices $-7965-$

\begin{tabular}{|c|c|c|c|c|c|}
\hline 65. & Solanum nigrum & Black night shade & $\begin{array}{c}\text { Fruit, Leaves, } \\
\text { Whole Plant }\end{array}$ & $\begin{array}{c}\text { Poultice, Infusion, } \\
\text { Extract }\end{array}$ & Herb, Wild \\
\hline 66. & Solanum surattense & Surattense nightshade & Fruit, Leaves, Roots & Decoction & Herb, Wild \\
\hline 67. & Withania somnifera & Ashwagandha & Whole Plant, Roots & Decoction & Herb, Wild \\
\hline 68. & Tamarix dioica & Saltcedar & Branches, Leaves & Powder & Shurb, Wild \\
\hline 69. & Grewia asiatica & Phalsa & Branches, Fruit & Decoction, Juice & $\begin{array}{c}\text { Tree, } \\
\text { Wild/Cultivated }\end{array}$ \\
\hline 70. & Corchorus aestuans & Jute mallow & Seeds, Whole Plant & Powder & Herb, Wild \\
\hline 71. & Tribulus camaldulensis & River red gum & $\begin{array}{c}\text { Seeds, Fruits, } \\
\text { Leaves }\end{array}$ & Powder, Infusion & Herb, Wild \\
\hline 72. & Scandix pectenveneris & Shepherd's needle & $\begin{array}{c}\text { Stems, Leaves, } \\
\text { Roots } \\
\end{array}$ & Powder, Decoction & Herb, Wild \\
\hline 73. & Rhazya stricta & Rhazya & $\begin{array}{c}\text { Stems, Leaves, } \\
\text { Fruits } \\
\end{array}$ & Extract & Shrub, Wild \\
\hline 74. & Phoenix dactylifera & Date palm & Leaves, Fruits & Extract, Decoction & Tree, Cultivated \\
\hline 75. & Calendula arvensis & Field marigold & Leaves, Flowers & Extract & Herb, Wild \\
\hline 76. & Conyza aegyptiaca & Canadian horseweed & Leaves, Flowers & Extract & Herb, Wild \\
\hline 77. & Bombax ceiba & $\begin{array}{c}\text { Cotton tree/red silk } \\
\text { cotton }\end{array}$ & Leaves, Stem, Seeds & Extract, Decoction & Herb, Wild \\
\hline 78. & Aloe vera & $\begin{array}{c}\text { Indian aloe/Chinese } \\
\text { aloe }\end{array}$ & Leaves, Gel & $\begin{array}{c}\text { Extract, Decoction, } \\
\text { Gel }\end{array}$ & $\begin{array}{c}\text { Shrub, } \\
\text { Wild/Cultivated }\end{array}$ \\
\hline 79. & Allium cepa & Onion & Leaves, Stem, Bulbs & Extract & Herb, Cultivated \\
\hline 80. & Azadirachta indica & Neem & Leaves & Extract, Decoction & $\begin{array}{c}\text { Herb, } \\
\text { Wild/Cultivated }\end{array}$ \\
\hline 81. & Althea rosea & Holyoke & $\begin{array}{c}\text { Leaves, Flowers, } \\
\text { Stems }\end{array}$ & Extract, Decoction & $\begin{array}{c}\text { Herb, } \\
\text { Wild/Cultivated }\end{array}$ \\
\hline 82. & Asparagus racemosus & Satawari & $\begin{array}{c}\text { Leaves, Flowers, } \\
\text { Stems }\end{array}$ & Extract, Decoction & Shrub, Wild \\
\hline 83. & Vinca major & Bigleaf periwinkle & Leaves, Seeds & Extract, Decoction & Shrub, Wild \\
\hline 84. & Cichorium intybus & Chicory & Leaves, Stem, Seeds & Extract, Decoction & $\begin{array}{c}\text { Weed, } \\
\text { Wild/Cultivated }\end{array}$ \\
\hline 85. & Fumaria indica & Fumitory & Leaves, Whole Plant & Extract & Weed, Wild \\
\hline 86. & Lepidium didymium & Swine cress & Leaves, Whole Plant & Extract & Herb, Wild \\
\hline 87. & Malvastrum coromandelianum & Red malvastrum & Leaves, Flowers & Decoction & Shrub, Wild \\
\hline 88. & Melilotus indica & Sweet clover & Leaves, Whole Plant & Decoction & Herb, Wild \\
\hline 89. & Micromeria biflora & Lemon scented thyme & Whole plant & Decoction & $\begin{array}{c}\text { Herb, } \\
\text { Wild/Cultivated }\end{array}$ \\
\hline 90. & Ranunculus muricatus & Scilly buttercup & $\begin{array}{l}\text { Whole plant, Fruit, } \\
\text { Leaves }\end{array}$ & Decoction & Herb, Wild \\
\hline 91. & Quercus incana & Bluejack oak & Leaves & Extract & Tree, Cultivated \\
\hline 92. & Berberis lyceum & Barberry & Leaves, Roots, Stem & Extract, Decoction & $\begin{array}{c}\text { Shrub, } \\
\text { Wild/Cultivated }\end{array}$ \\
\hline 93. & Debregeasia salicifolia & Debregeasia & Leaves & Powder & Shrub, Wild \\
\hline 94. & Dodonaea viscosa purpurea & $\begin{array}{c}\text { Broad leaf } \\
\text { hopbush/candle wood }\end{array}$ & $\begin{array}{c}\text { Leaves, Flowers, } \\
\text { Stem }\end{array}$ & $\begin{array}{c}\text { Powder, Decoction, } \\
\text { Oil, Extract }\end{array}$ & Shrub, Wild \\
\hline 95. & Hypericum oblongifolium & Pendant St. John's wort & Leaves, Fruits & Decoction, Extract & $\begin{array}{c}\text { Shrub, } \\
\text { Wild/Cultivated }\end{array}$ \\
\hline 96. & Indigofera heterantha & Himalayan indigo & Leaves, Fruits & Powder, Extract & Shrub, Wild \\
\hline 97. & Jasminum officinale & Common jasmine & Leaves, Flowers & Decoction, Extract & $\begin{array}{c}\text { Shrub, } \\
\text { Wild/Cultivated }\end{array}$ \\
\hline 98 & Lonicera quinquelocularis & $\begin{array}{r}\text { Translucent } \\
\text { honeysuckle }\end{array}$ & Leaves, Flowers & Extract & Shrub, Wild \\
\hline 99. & Zanthoxylum armatum & Winged prickly ash & Whole Plant, Leaves & $\begin{array}{c}\text { Extract, Decoction, } \\
\text { Powder }\end{array}$ & Shrub, Cultivated \\
\hline 100 & Duchesnea indica & Indian strawberry & Whole plant & Extract, Decoction, & Herb, Wild \\
\hline
\end{tabular}




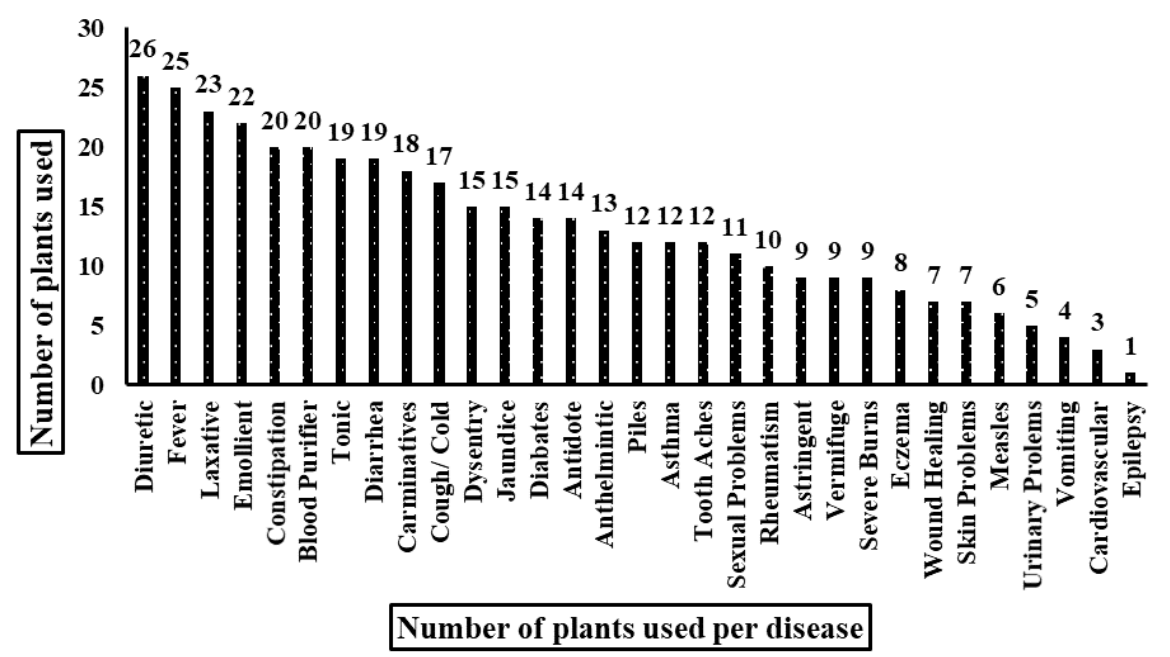

Figure 5. Number of plants used for treatment of diseases studied from 3 selected sites of Gujranwala region

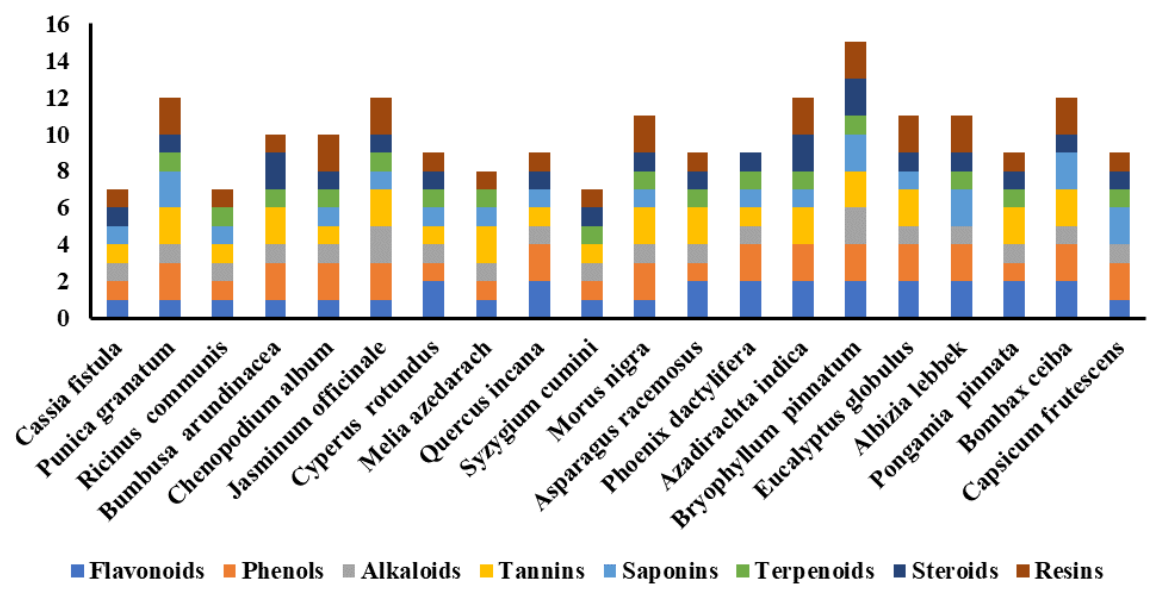

Figure 6. Comparison of phytochemicals present in selected species studied in 3 selected sites of Gujranwala region

\section{Quantitative indices}

Medicinal values of different plants belonging to different families are present in Table 4, along with their common name, scientific name, families, quantitative indices viz., UV, FC, RFC, UR, RIL, FL, CFL and therapeutic uses. Plants of different types were found in the study area that has different therapeutic uses. They were quantitatively analyzed by using different formulas. Factor of informant consensus can be particularly useful to select the categories of diseases for which the species can traditionally are used. The relative importance level (RIL) presents the level of prominence of each species in a study site. Fidelity level (FL) of species uses, i.e. the ratio between the number of informants who independently cite the use of a species for the same major purposes use reports (URs) and the total number of informants who mentioned the plant for any use. Corrected fidelity level (CFL) of plant species were used as correction factor to accurately rank the plant species with different FL and RIL values. CFL was derived from FL, by multiplying FL with RIL values. 
Table 4. Inventory of plant species, their quantitative analysis, therapeutic uses and reports of Gujranwala area

\begin{tabular}{|c|c|c|c|c|c|c|c|c|c|c|c|}
\hline S.No & Common name & Scientific name and voucher specimen & Family & UV & FCI & RFC & UR & RIL & FL & CFL & Therapeutic uses \\
\hline 1. & Bahar Bail & $\begin{array}{l}\text { Convolvulus album } \mathrm{L} . \\
\text { UOG-001005 }\end{array}$ & Convolvulaceae & 0.89 & 27 & 0.11 & 15 & 0.89 & 50 & 25 & $\begin{array}{c}\text { Emollient, anthelminthic, urinary tract infections, } \\
\text { increasing bile production }\end{array}$ \\
\hline 2. & Zakhm-e-hayat & $\begin{array}{l}\text { Bryophyllum pinnatum } \mathrm{L} . \\
\text { UOG-001006 }\end{array}$ & Crassulaceae & 0.71 & 11 & 0.02 & 15 & 0.78 & 70 & 35 & $\begin{array}{c}\text { Emollient, analgesic, carminative, vomiting, } \\
\text { diarrhea }\end{array}$ \\
\hline 3. & Bhung & $\begin{array}{l}\text { Cannabis sativa } \mathrm{L} . \\
\text { UOG-001007 }\end{array}$ & Cannabinaceae & 0.76 & 22 & 0.13 & 18 & 0.81 & 89.2 & 47 & Tonic, relaxing purposes \\
\hline 4. & Bathu & $\begin{array}{l}\text { Chenopodium album } \mathrm{L} . \\
\text { UOG-001008 }\end{array}$ & Chenopodiaceae & 0.67 & 14 & 0.03 & 19 & 0.56 & 77 & 38 & Laxative, urinary problems, sexual diseases \\
\hline 5. & Gorakh Paan & $\begin{array}{l}\text { Heliotropium strigosum } \mathrm{L} . \\
\text { UOG-001009 }\end{array}$ & Boraginaceae & 0.87 & 12 & 0.01 & 19 & 0.98 & 34 & 26 & Diuretic, blood purifier, liver tonic \\
\hline 6. & Chota Dathoora & $\begin{array}{l}\text { Xanthium strumarium L. } \\
\text { UOG-001010 }\end{array}$ & Asteraceae & 0.65 & 16 & 0.15 & 17 & 0.89 & 64 & 34 & Sedative, diuretic, eczema \\
\hline 7. & Kulfa & $\begin{array}{l}\text { Trichodesma indicum } \text { L. R. Br. } \\
\text { UOG-001011 }\end{array}$ & Boraginaceae & 0.98 & 15 & 0.16 & 11 & 0.67 & 78 & 44 & Antidote, diuretic, dysentery, fever, diarrhea \\
\hline 8. & Tumma & $\begin{array}{l}\text { Citrullus colocynthis } \mathrm{L} . \\
\text { UOG-001012 }\end{array}$ & Cucurbitaceae & 0.10 & 22 & 0.12 & 13 & 0.78 & 45 & 24 & $\begin{array}{l}\text { Purgative, amenorrhea, constipation, stomach } \\
\text { aches, jaundice }\end{array}$ \\
\hline 9. & Bhtha & $\begin{array}{l}\text { Benincasa hispida } \mathrm{L} . \\
\text { UOG-001013 }\end{array}$ & Cucurbitaceae & 0.45 & 25 & 0.16 & 15 & 0.65 & 46.35 & 20 & Laxative, diuretic, tonic, anthelminthic, epilepsy \\
\hline 10. & Jangli Khaboz & $\begin{array}{l}\text { Cucumus melo L. } \\
\text { UOG-001014 }\end{array}$ & Cucurbitaceae & 0.76 & 10 & 0.14 & 12 & 0.78 & 78 & 40 & Laxative, dysuria, leucorrhea, eczema \\
\hline 11. & Kaddu & $\begin{array}{l}\text { Cucurbita pepo L. } \\
\text { UOG-001015 }\end{array}$ & Cucurbitaceae & 0.56 & 20 & 0.16 & 12 & 0.56 & 25 & 10 & Anthelminthic, emollient \\
\hline 12. & Neeli Taar & $\begin{array}{l}\text { Cuscuta reflexa R. } \\
\text { UOG-001016 }\end{array}$ & Cuscutaceae & 0.67 & 27 & 0.17 & 15 & 0.89 & 28 & 13 & Rheumatism, anti-diabetic, toothache \\
\hline 13. & Spodhodal & $\begin{array}{l}\text { Euphorbia hirta L. } \\
\text { UOG-001017 }\end{array}$ & Euphorbiaceae & 0.45 & 17 & 0.19 & 18 & 0.87 & 30 & 18 & Asthma, applied on injuries \\
\hline 14. & Dhodal & $\begin{array}{l}\text { Euphorbia heliscopia L. } \\
\text { UOG-001018 }\end{array}$ & Euphorbiaceae & 0.87 & 27 & 0.023 & 19 & 0.67 & 40 & 39 & Purgative, skin eruptions \\
\hline 15. & Arnd & $\begin{array}{l}\text { Ricinus communis } \mathrm{L} \text {. } \\
\text { UOG-001019 }\end{array}$ & Euphorbiaceae & 0.89 & & 0.07 & 21 & 0.87 & 60 & 35 & Regulate menses, scabies, obesity \\
\hline 16. & Deela & $\begin{array}{l}\text { Cyperus rotundus } \mathrm{L} . \\
\quad \mathrm{UOG}-001020\end{array}$ & Cyperaceae & 0.76 & 19 & 0.30 & 22 & 0.78 & 40 & 19 & $\begin{array}{c}\text { Diuretic, vermifuge, tonic for animals, diarrhea, } \\
\text { pneumonia, children dysentery, antidote }\end{array}$ \\
\hline
\end{tabular}




\begin{tabular}{|c|c|c|c|c|c|c|c|c|c|c|c|}
\hline S.No & Common name & Scientific name and voucher specimen & Family & $\mathbf{U V}$ & FCI & RFC & UR & RIL & FL & CFL & Therapeutic uses \\
\hline 17. & Turkhrai & $\begin{array}{l}\text { Indigofera linifolia } \mathrm{L} \text {. } \\
\text { UOG-001021 }\end{array}$ & Papilionaceae & 0.89 & 10 & 0.078 & 10 & 0.89 & 56 & 30 & Emollient, febrile skin problems \\
\hline 18. & Shishum & $\begin{array}{l}\text { Dalbergia sissoo L. } \\
\quad \text { UOG-001022 }\end{array}$ & Papilionaceae & 0.13 & 29 & 0.54 & 20 & 0.99 & 64 & 34 & Emollient, emetic, dysentery, fever, pyorrhea \\
\hline 19. & Sukhchain & $\begin{array}{l}\text { Pongamia pinnata L. } \\
\text { UOG-001023 }\end{array}$ & Papilionaceae & 0.24 & 10 & 0.67 & 22 & 0.78 & 84 & 67 & Carminative, vermifuge, rheumatism, toothache \\
\hline 20. & Sann & $\begin{array}{l}\text { Crotolaria burhia } \mathrm{L} . \\
\text { UOG-001024 }\end{array}$ & Papilionaceae & 0.65 & 15 & 0.12 & 52 & 0.87 & 37 & 20 & Diuretic, blood purifier \\
\hline 21. & Phuwa & $\begin{array}{l}\text { Alhagi maurorum L. } \\
\text { UOG-001025 }\end{array}$ & Papilionaceae & 0.23 & 25 & 0.15 & 26 & 0.78 & 90 & 65 & Piles, constipation \\
\hline 22. & Stianasi & $\begin{array}{l}\text { Argemone mexicana } \mathrm{L} . \\
\text { UOG-001026 }\end{array}$ & Papaveraceae & 0.67 & 32 & 0.12 & 25 & 0.98 & 56 & 37 & $\begin{array}{c}\text { Laxative, effective against premature ejaculation, } \\
\text { spermatorrhea }\end{array}$ \\
\hline 23. & Khbal Kha & $\begin{array}{l}\text { Cynodon dactylon } \mathrm{L} . \\
\text { UOG-001027 }\end{array}$ & Poaceae & 0.87 & 27 & 0.16 & 15 & 0.56 & 73 & 49 & Purgative, epistasis, dysentery, blood purifier \\
\hline 24. & Javi & $\begin{array}{l}\text { Avena sativa } \mathrm{L} . \\
\text { UOG-001028 }\end{array}$ & Poaceae & 0.89 & 29 & 0.16 & 13 & 0.89 & 72 & 50 & Antispasmodic, liver tonic, diuretic \\
\hline 25. & Baans & $\begin{array}{l}\text { Bumbusa arundinacea } \mathrm{L} . \\
\text { UOG-001029 }\end{array}$ & Poaceae & 0.67 & 28 & 0.16 & 17 & 0.76 & 40 & 10 & $\begin{array}{l}\text { Carminative, cold, flu, fever, effective against, } \\
\text { ringworms burns }\end{array}$ \\
\hline 26. & Gulab & $\begin{array}{l}\text { Rosa indica } \mathrm{L} . \\
\text { UOG-001030 }\end{array}$ & Rosaceae & 0.77 & 21 & 0.19 & 16 & 0.56 & 37 & 14 & $\begin{array}{l}\text { Emollient, laxative, anthelminthic, diuretic, } \\
\text { constipation }\end{array}$ \\
\hline 27. & Bair & $\begin{array}{l}\text { Zizyphus nummularia } \mathrm{L} . \\
\text { UOG-001031 }\end{array}$ & Rhamnaceae & 0.60 & 27 & 0.20 & 29 & 0.78 & 48 & 27 & $\begin{array}{l}\text { Purgative, emollient, antiseptic, measles, } \\
\text { constipation }\end{array}$ \\
\hline 28. & Anaar & $\begin{array}{l}\text { Punica granatum L. } \\
\text { UOG-001032 }\end{array}$ & Punicaceae & 0.83 & 26 & 0.23 & 17 & 0.87 & 47 & 19 & Dysentery, pyorrhea, blood purifier \\
\hline 29. & Kulfa & $\begin{array}{l}\text { Portulaca oleracea L. } \\
\text { UOG-001033 }\end{array}$ & Portulacaceae & 0.34 & 25 & 0.34 & 19 & 0.450 & 45 & 13 & Jaundice, liver and spleen disorders \\
\hline 30. & Desi Kulfa & $\begin{array}{l}\text { Portulaca quadrifida } \mathrm{L} \text {. } \\
\text { UOG-001034 }\end{array}$ & Portulacaceae & 0.78 & 21 & 0.13 & 29 & 0.78 & 47 & 15 & Vermifuge, piles, cold, flu, constipation \\
\hline 31. & Water Hyacinth & $\begin{array}{l}\text { Eichhornia crassipes L. } \\
\text { UOG-001035 }\end{array}$ & Pontederiaceae & 0.56 & 26 & 0.167 & 22 & 0.98 & 26 & 12 & Emollient, blood purifier \\
\hline 32. & Droonk & $\begin{array}{l}\text { Polygonum plebium L. } \\
\text { UOG-001036 }\end{array}$ & Polygonaceae & 0.87 & 12 & 0.121 & 10 & 0.78 & 30 & 16 & Eczema, heartburns \\
\hline 33. & Sura & $\begin{array}{l}\text { Hibiscus rosasinensis } \mathrm{L} . \\
\quad \text { UOG-001037 }\end{array}$ & Malvaceae & 0.56 & 23 & 0.321 & 15 & 0.98 & 38 & 26 & $\begin{array}{l}\text { Laxative, effective against burning sensation in } \\
\text { the urine, constipation }\end{array}$ \\
\hline
\end{tabular}

APPLIED ECOLOGY AND ENVIRONMENTAL RESEARCH 18(6):7953-7979.

http://www.aloki.hu • ISSN 15891623 (Print) • ISSN 17850037 (Online)

DOI: http://dx.doi.org/10.15666/aeer/1806_79537979

(c) 2020, ALÖKI Kft., Budapest, Hungary 


\begin{tabular}{|c|c|c|c|c|c|c|c|c|c|c|c|}
\hline S.No & Common name & Scientific name and voucher specimen & Family & $\mathbf{U V}$ & FCI & RFC & UR & RIL & FL & CFL & Therapeutic uses \\
\hline 34. & Pelae & $\begin{array}{l}\text { Abutilon indicum L. } \\
\text { UOG-001038 }\end{array}$ & Malvaceae & 0.78 & 20 & 0.176 & 170 & 0.89 & 67 & 56 & Purgative, piles \\
\hline 35. & Sunchal & $\begin{array}{l}\text { Marva parviflora } \mathrm{L} . \\
\text { UOG-001039 }\end{array}$ & Malvaceae & 0.64 & 27 & 0.321 & 190 & 0.99 & 98 & 78 & Laxative, cold, flu, cough, fever \\
\hline 36. & Dhreek & $\begin{array}{l}\text { Melia azedarach L. } \\
\text { UOG-001040 }\end{array}$ & Malvaceae & 0.76 & 26 & 0.165 & 130 & 0.66 & 36 & 29 & Emollient, laxative, antidote, blood purifier \\
\hline 37. & Podeena & $\begin{array}{l}\text { Mentha spicata } \mathrm{L} . \\
\text { UOG-001041 }\end{array}$ & Lamiaceae & 0.54 & 25 & 0.178 & 190 & 0.78 & 48 & 20 & Carminative, diarrhea \\
\hline 38. & Gul-e-Asar & $\begin{array}{l}\text { Mirabilis jalapa L. } \\
\text { UOG-001042 }\end{array}$ & Nyctaginaceae & 0.44 & 24 & 0.154 & 280 & 0.88 & 40 & 10 & Purgative, Skin eruptions, constipation, hepatitis \\
\hline 39. & Jamun & $\begin{array}{l}\text { Syzygium cumini L. R. } \\
\text { UOG-001043 }\end{array}$ & Myrtaceae & 0.57 & 19 & 0.12 & 380 & 0.67 & 20 & 10 & Diabetes \\
\hline 40. & Safaida & $\begin{array}{l}\text { Eucalyptus globulus L. R. } \\
\text { UOG-001044 }\end{array}$ & Myrtaceae & 0.89 & 17 & 0.16 & 170 & 0.98 & 89 & 56 & Cold, flu, fever, sore throat \\
\hline 41. & Kikar & $\begin{array}{l}\text { Acacia nilotica L. } \\
\text { UOG-001045 }\end{array}$ & Mimosaceae & 0.98 & 14 & 0.156 & 270 & 0.78 & 54 & 22 & Stops premature ejaculation, helps in digestion \\
\hline 42. & Shirin & $\begin{array}{l}\text { Albizia lebbek } \mathrm{L} . \\
\text { UOG-001046 }\end{array}$ & Mimosaceae & 0.97 & 21 & 0.12 & 28 & 0.98 & 39 & 18 & Diuretic, asthma, blood purifier \\
\hline 43. & Amaltas & $\begin{array}{l}\text { Cassia fistula L. } \\
\text { UOG-001047 }\end{array}$ & Mimosaceae & 0.79 & 24 & 0.21 & 26 & 0.67 & 56 & 27 & Diarrhea \\
\hline 44. & Phulae & $\begin{array}{c}\text { Acacia modesta } \mathrm{L} . \\
\text { UOG-001048 }\end{array}$ & Mimosaceae & 0.94 & 26 & 0.16 & 37 & 0.66 & 54 & 26 & Carminative \\
\hline 45. & Bohrh & $\begin{array}{l}\text { Ficus benghalensis } \mathrm{L} . \\
\text { UOG-001049 }\end{array}$ & Moraceae & 0.56 & 20 & 0.16 & 34 & 0.67 & 38 & 21 & $\begin{array}{c}\text { Stop premature ejaculation, syphilis, gonorrhea, } \\
\text { provides sexual strength }\end{array}$ \\
\hline 46. & Kala Toot & $\begin{array}{l}\text { Morus nigra L. } \\
\text { UOG-001050 }\end{array}$ & Moraceae & 0.87 & 30 & 0.21 & 30 & 0.87 & 30 & 16 & $\begin{array}{l}\text { Carminative, blood purifier, sore throat, cold, } \\
\text { cough }\end{array}$ \\
\hline 47. & Khktl & $\begin{array}{l}\text { Oxalis corniculata } \mathrm{L} \text {. } \\
\text { UOG- } 001051\end{array}$ & Oxalidaceae & 0.37 & 20 & 0.71 & 29 & 0.67 & 40 & 20 & Emollient, spermatorrhea \\
\hline 48. & Saunf & $\begin{array}{l}\text { Foeniculum vulgare } \mathrm{L} . \\
\text { UOG-001052 }\end{array}$ & Apiaceae & 0.44 & 18 & 0.13 & 37 & 0.87 & 89 & 65 & $\begin{array}{l}\text { Carminative, purgative, diuretic, laxative, gas } \\
\text { trouble, enhances eye sight }\end{array}$ \\
\hline 49. & Itst & $\begin{array}{c}\text { Trianthema portulacastrum } \mathrm{L} . \\
\text { UOG-001053 } \\
\end{array}$ & Aizoaceae & 0.76 & 19 & 0.34 & 37 & 0.56 & 65 & 47 & $\begin{array}{l}\text { Anthelmintic, diuretic, asthma, liver infection, } \\
\text { jaundice }\end{array}$ \\
\hline 50. & Baykr & $\begin{array}{l}\text { Justicia adhatoda } \mathrm{L} . \\
\text { UOG-001054 }\end{array}$ & Acanthaceae & 0.67 & 15 & 0.122 & 35 & 0.23 & 98 & 88 & Abortifacient, cold, cough, flu, toothache, fever \\
\hline
\end{tabular}

APPLIED ECOLOGY AND ENVIRONMENTAL RESEARCH 18(6):7953-7979.

http://www.aloki.hu • ISSN 15891623 (Print) • ISSN 17850037 (Online)

DOI: http://dx.doi.org/10.15666/aeer/1806_79537979

(c) 2020, ALÖKI Kft., Budapest, Hungary 


\begin{tabular}{|c|c|c|c|c|c|c|c|c|c|c|c|}
\hline S.No & Common name & Scientific name and voucher specimen & Family & UV & FCI & RFC & UR & RIL & FL & CFL & Therapeutic uses \\
\hline 51. & Thoom & $\begin{array}{l}\text { Allium sativum } \mathrm{L} \text {. } \\
\text { UOG-001055 }\end{array}$ & Amaryllidaceae & 0.19 & 16 & 0.33 & 28 & 0.65 & 70 & 45 & $\begin{array}{l}\text { Lowering blood pressure, cardiac problems. } \\
\text { Respiratory tract infection, hypertension }\end{array}$ \\
\hline 52. & Kanair & $\begin{array}{l}\text { Nerium oleander } \mathrm{L} . \\
\text { UOG-001056 }\end{array}$ & Apocynaceae & 0.18 & 35 & 0.78 & 45 & 0.67 & 54 & 30 & Abortions, toothache, earache \\
\hline 53. & Ghunar & $\begin{array}{l}\text { Amaranthus viridis L. R. Br. } \\
\text { UOG-001057 }\end{array}$ & Amaranthaceae & 0.67 & 34 & 0.67 & 26 & 0.78 & 89 & 67 & Carminative, emollient, rheumatism \\
\hline 54. & Puthkanda & $\begin{array}{c}\text { Achyranthes aspera L. R. Br. } \\
\text { UOG-001058 }\end{array}$ & Amaranthaceae & 0.76 & 27 & 0.76 & 19 & 0.98 & 87 & 56 & Pyorrhea, cold, flu, fever, pneumonia leprosy \\
\hline 55. & Desi Aak & $\begin{array}{l}\text { Calotropis procera L. R. Br. } \\
\text { UOG-001059 }\end{array}$ & Asclepiadaceae & 0.18 & 24 & 0.007 & 27 & 0.67 & 67 & 35 & $\begin{array}{c}\text { Expectorant, antidote, scabies, rheumatism, } \\
\text { dysentery }\end{array}$ \\
\hline 56. & Chonga & $\begin{array}{l}\text { Caralluma edulis L. R. Br. } \\
\text { UOG-001060 }\end{array}$ & Asclepiadaceae & 0.18 & 18 & 0.0675 & 29 & 0.54 & 50 & 25 & Anthelmintic, diuretic, diabetes \\
\hline 57. & Partha & $\begin{array}{l}\text { Parthenium hysterophorus L. R. Br. } \\
\text { UOG-001061 }\end{array}$ & Asteraceae & 1.05 & 17 & 0.67 & 26 & 0.67 & 78 & 44 & Laxatives, diabetes, regulate bowl movements \\
\hline 58. & Chaou & $\begin{array}{l}\text { Artemisia scoparia } \mathrm{L} . \\
\text { UOG-001062 }\end{array}$ & Asteraceae & 0.37 & 27 & 0.76 & 17 & 0.87 & 56 & 35 & Purgatives, antidote, malaria \\
\hline 59. & Bhangra & $\begin{array}{l}\text { Eclipta prostrate } \mathrm{L} . \\
\text { UOG-001063 }\end{array}$ & Asteraceae & 0.80 & 28 & 0.89 & 14 & 0.78 & 78 & 45 & Emetic, jaundice \\
\hline 60. & Pholi & $\begin{array}{c}\text { Carthamus oxyacantha } \mathrm{L} . \\
\text { UOG-001064 }\end{array}$ & Asteraceae & 0.90 & 35 & 0.003 & 2019 & 0.76 & 45 & 20 & Ulcer, jaundice \\
\hline 61. & Hund & $\begin{array}{c}\text { Taraxacum officinale L. R. } \\
\text { UOG-001065 }\end{array}$ & Asteraceae & 0.79 & 12 & 0.330 & 22 & 0.89 & 47 & 26 & Constipation, diabetes, antidote \\
\hline 62. & Pelo & $\begin{array}{l}\text { Salvadora oleoides L. } \\
\text { UOG-001066 }\end{array}$ & Salvadoraceae & 0.76 & 29 & 0.012 & 21 & 0.87 & 30.78 & 12 & Toothache, tonic \\
\hline 63. & Siah Dahtora & $\begin{array}{l}\text { Datura innoxia L. } \\
\text { UOG-001067 }\end{array}$ & Solanaceae & 0.29 & 12 & 0.0456 & 25 & 0.90 & 67 & 33 & Sedative, stops premature ejaculation \\
\hline 64. & Surkh Mirch & $\begin{array}{l}\text { Capsicum frutescens } \mathrm{L} . \\
\text { UOG-001068 }\end{array}$ & Solanaceae & 1.08 & 22 & 0.054 & 40 & 0.87 & 56 & 31 & Dyspepsia \\
\hline 65. & Kaanch Mainch & $\begin{array}{l}\text { Solanum nigrum L. } \\
\text { UOG-001069 }\end{array}$ & Solanaceae & 1.02 & 24 & 0.067 & 45 & 0.99 & 49 & 28 & Laxative, jaundice, hepatitis, obesity \\
\hline 66. & Mookri & $\begin{array}{l}\text { Solanum surattense L. } \\
\text { UOG-001070 }\end{array}$ & Solanaceae & 0.67 & 25 & 0.05 & 15 & 0.34 & 36 & 29 & Rheumatism, fever, asthma \\
\hline 67. & Aksn & $\begin{array}{l}\text { Withania somnifera L. R. } \\
\text { UOG-001071 }\end{array}$ & Solanaceae & 1.0 & 21 & 0.60 & 19 & 0.76 & 38 & 29 & Dysentery, diarrhea \\
\hline
\end{tabular}

APPLIED ECOLOGY AND ENVIRONMENTAL RESEARCH 18(6):7953-7979.

http://www.aloki.hu • ISSN 15891623 (Print) • ISSN 17850037 (Online)

DOI: http://dx.doi.org/10.15666/aeer/1806_79537979

○ 2020, ALÖKI Kft., Budapest, Hungary 


\begin{tabular}{|c|c|c|c|c|c|c|c|c|c|c|c|}
\hline S.No & Common name & Scientific name and voucher specimen & Family & UV & FCI & RFC & UR & RIL & FL & CFL & Therapeutic uses \\
\hline 68. & Rukh & $\begin{array}{l}\text { Tamarix dioica } \mathrm{L} . \\
\text { UOG-001072 }\end{array}$ & Tamaricaceae & 0.56 & 26 & 0.19 & 16 & 0.99 & 98 & 78 & Piles, diarrhea \\
\hline 69. & Falsa & $\begin{array}{l}\text { Grewia asiatica } \mathrm{L} . \\
\text { UOG-001073 } \\
\end{array}$ & Tiliaceae & 0.98 & 27 & 0.19 & 15 & 0.47 & 36 & 25 & Diuretic, constipation emetic, blood purifier \\
\hline 70. & Jute & $\begin{array}{l}\text { Corchorus aestuans } \mathrm{L} . \\
\text { UOG-001074 }\end{array}$ & Tiliaceae & 0.76 & 19 & 0.19 & 13 & 0.36 & 40 & 20 & Pneumonia \\
\hline 71. & Pakhra & $\begin{array}{c}\text { Tribulus camaldulensis L. } \\
\text { UOG-001075 }\end{array}$ & Zygophyllaceae & 0.67 & 19 & 0.167 & 18 & 0.98 & 93 & 67 & Remove gall bladder and kidney stones \\
\hline 72. & Ziri & $\begin{array}{l}\text { Scandix pectenveneris } \mathrm{L} \text {. } \\
\text { UOG-001076 }\end{array}$ & Amaranthaceae & 0.54 & 24 & 0.7 & 14 & 0.67 & 27 & 12 & Astringent, palpitations, blood coagulant \\
\hline 73. & Rangobul & $\begin{array}{l}\text { Rhazya stricta } \mathrm{L} . \\
\text { UOG-001077 }\end{array}$ & Apocynaceae & 0.23 & 23 & 0.778 & 17 & 0.56 & 39 & 22 & Antihypertensive \\
\hline 74. & Khajoor & $\begin{array}{l}\text { Phoenix dactylifer L. } \\
\text { UOG-001078 }\end{array}$ & Arecaceae & 0.46 & 18 & 0.56 & 15 & 0.87 & 20 & 06 & Astringent, gonorrhea, abdominal pains \\
\hline 75. & Gul-e-Sharf & $\begin{array}{l}\text { Calendula arvensis } \mathrm{L} . \\
\text { UOG-001079 }\end{array}$ & Asteraceae & 0.65 & 27 & 0.655 & 20 & 0.67 & 48 & 20 & Severe pains \\
\hline 76. & Gul-e-Hozah & $\begin{array}{l}\text { Conyza aegyptiaca } \mathrm{L} . \\
\text { UOG-001080 }\end{array}$ & Asteraceae & 0.66 & 15 & 0.006 & 22 & 0.87 & 49 & 29 & Diarrhea, fever, toothache, earache \\
\hline 77. & Sumbal & $\begin{array}{l}\text { Bombax ceiba } \mathrm{L} . \\
\text { UOG-001081 }\end{array}$ & Asteraceae & 0.78 & 27 & 0.45 & 25 & 0.78 & 46 & 28 & Sericulture \\
\hline 78. & Kawar Gandal & $\begin{array}{l}\text { Aloe vera } \mathrm{L} . \\
\text { UOG-001082 }\end{array}$ & Asphodelaceae & 0.53 & 26 & 0.65 & 27 & 0.89 & 45 & 20 & Acne, pimples \\
\hline 79. & Ganda & $\begin{array}{l}\text { Allium cepa } \mathrm{L} \text {. } \\
\text { UOG-001083 }\end{array}$ & Amaryllidaceae & 0.83 & 14 & 0.34 & 29 & 0.87 & 90 & 67 & Disinfectant, earache, cardiovascular disorders \\
\hline 80. & Neem & $\begin{array}{l}\text { Azadirachta indica } \mathrm{L} . \\
\text { UOG-001084 }\end{array}$ & Meliaceae & 0.67 & 20 & 0.56 & 27 & 0.78 & 87 & 50 & Joints pain, malaria \\
\hline 81. & Gul-e-Khairu & $\begin{array}{l}\text { Althea rosea L. R. } \\
\text { UOG-001085 }\end{array}$ & Malvaceae & 0.91 & 18 & 0.87 & 24 & 0.76 & 45 & 27 & $\begin{array}{l}\text { Laxative, expectorant, demulcent, emollient, } \\
\text { chest pain }\end{array}$ \\
\hline 82. & Moosli Safaid & $\begin{array}{l}\text { Asparagus racemosus } \mathrm{L} \text {. } \\
\text { UOG-001086 }\end{array}$ & Asparagaceae & 0.35 & 27 & 0.16 & 37 & 0.89 & 47 & 28 & Measles, liver tonic, heal wounds, asthma \\
\hline 83. & Periwinkle & $\begin{array}{l}\text { Vinca major L. } \\
\text { UOG-001087 }\end{array}$ & Apocynaceae & 0.87 & 22 & 0.02 & 20 & 0.65 & 49 & 20 & Diarrhea, cancer, diabetes \\
\hline 84. & Kasni & $\begin{array}{l}\text { Cichorium intybus L. } \\
\text { UOG-001088 }\end{array}$ & Asteraceae & 0.17 & 25 & 0.34 & 34 & 0.78 & 29 & 13 & Diuretic, stimulant \\
\hline
\end{tabular}

APPLIED ECOLOGY AND ENVIRONMENTAL RESEARCH 18(6):7953-7979.

http://www.aloki.hu • ISSN 15891623 (Print) • ISSN 17850037 (Online)

DOI: http://dx.doi.org/10.15666/aeer/1806_79537979

○ 2020, ALÖKI Kft., Budapest, Hungary 


\begin{tabular}{|c|c|c|c|c|c|c|c|c|c|c|c|}
\hline S.No & Common name & Scientific name and voucher specimen & Family & $\mathbf{U V}$ & FCI & RFC & UR & RIL & FL & CFL & Therapeutic uses \\
\hline 85 & Paprra & $\begin{array}{l}\text { Fumaria indica } \mathrm{L} . \\
\text { UOG-001089 }\end{array}$ & Papaveraceae & 0.82 & 27 & 0.65 & 24 & 0.87 & 39 & 17 & Fever, asthma \\
\hline 86. & Kurly Cress & $\begin{array}{l}\text { Lepidium didymium } \mathrm{L} . \\
\text { UOG-001090 }\end{array}$ & Brassicaceae & 0.27 & 12 & 0.034 & 27 & 0.89 & 38 & 17 & Antipyretic \\
\hline 87. & Bariar & $\begin{array}{l}\text { Malvastrum coromandelianum } \mathrm{L} . \\
\text { UOG-001091 }\end{array}$ & Malvaceae & 0.2 & 34 & 0.560 & 27 & 0.98 & 30 & 19 & Aphrodisiac, wound healing, fever \\
\hline 88. & Singi & $\begin{array}{l}\text { Melilotus indica } \mathrm{L} . \\
\text { UOG-001092 }\end{array}$ & Fabaceae & 0.67 & 26 & 0.006 & 27 & 0.88 & 37 & 29 & Abdominal pain, diabetes, dysentery, diarrhea \\
\hline 89. & Boine & $\begin{array}{l}\text { Micromeria biflora } \mathrm{L} . \\
\text { UOG-001093 }\end{array}$ & Lamiaceae & 0.83 & 18 & 0.007 & 5 & 0.98 & 38 & 20 & Diuretic, vomiting, constipation, headache \\
\hline 90. & Kor-Kandoli & $\begin{array}{l}\text { Ranunculus muricatus } \mathrm{L} \text {. } \\
\text { UOG-001094 }\end{array}$ & Rananculaceae & 0.18 & 27 & 0.070 & 18 & 0.78 & 37 & 27 & $\begin{array}{l}\text { Laxative, antidote, cough, remove tumors and } \\
\text { bursts }\end{array}$ \\
\hline 91. & Erian & $\begin{array}{l}\text { Quercus incana } \mathrm{L} . \\
\text { UOG-001095 }\end{array}$ & Fagaceae & 0.67 & 37 & 0.54 & 10 & 0.65 & 38 & 20 & Hemorrhagic septicemia, joint pain \\
\hline 92. & Komal & $\begin{array}{l}\text { Berberis lyceum L. } \\
\text { UOG-001096 }\end{array}$ & Berberidaceae & 0.54 & 24 & 0.006 & 2938 & 0.78 & 49 & 29 & $\begin{array}{l}\text { bone fractures, jaundice, wound healing, } \\
\text { dyspepsia, hypertension }\end{array}$ \\
\hline 93. & Sindari & $\begin{array}{l}\text { Debregeasia salicifolia } \mathrm{L} . \\
\text { UOG-001097 }\end{array}$ & Urticaceae & 0.48 & 18 & 0.654 & 30 & 0.87 & 50 & 25 & Febrifuge, jaundice \\
\hline 94. & Sanatha & $\begin{array}{c}\text { Dodonaea viscosa purpurea } \mathrm{L} \text {. } \\
\text { UOG-001098 }\end{array}$ & Sapindaceae & 0.95 & 10 & 0.67 & 29 & 0.76 & 48 & 30 & $\begin{array}{c}\text { Anthelmintic, healing burns, wounds and bruises } \\
\text { astringent, blood purifier, paralysis }\end{array}$ \\
\hline 95. & Pinli & $\begin{array}{l}\text { Hypericum oblongifolium } \mathrm{L} . \\
\text { UOG-001099 }\end{array}$ & Hypericaceae & 0.67 & 20 & 0.76 & 16 & 0.89 & 37 & 20 & Blood pressure, ulcer \\
\hline 96. & Hiran Charri & $\begin{array}{l}\text { Indigofera heterantha } \mathrm{L} \text {. } \\
\text { UOG-001100 }\end{array}$ & Fabaceae & 0.9 & 37 & 0.34 & 10 & 0.98 & 40 & 15 & Vermifuge, hepatitis \\
\hline 97. & Chambeli & $\begin{array}{l}\text { Jasminum officinale L. } \\
\text { UOG-001101 }\end{array}$ & Oleaceae & 0.36 & 22 & 0.543 & 20 & 0.76 & 57 & 28 & Anti-inflammatory, ringworms \\
\hline 98. & Phutt & $\begin{array}{l}\text { Lonicera quinquelocularis L. } \\
\text { UOG-001102 }\end{array}$ & Caprifoliaceae & 0.87 & 20 & 0.001 & 37 & 0.99 & 69 & 37 & $\begin{array}{l}\text { Improve vision, remove cataract, ophthalmic } \\
\text { agent, wound healing }\end{array}$ \\
\hline 99. & Timbar & $\begin{array}{l}\text { Zanthoxylum armatum L. } \\
\text { UOG-001103 }\end{array}$ & Rutaceae & 0.10 & 12 & 0.004 & 19 & 0.99 & 46 & 21 & Carminative, dyspepsia, stomach pains, piles \\
\hline 100. & Surkh Akhra & $\begin{array}{l}\text { Duchesnea indica L. } \\
\text { UOG-001104 }\end{array}$ & Rosaceae & 0.45 & 23 & 0.760 & 12 & 0.45 & 48 & 23 & $\begin{array}{c}\text { Diuretic, astringent, laxative, sore throat, nerve } \\
\text { tonic }\end{array}$ \\
\hline
\end{tabular}

Informant consensus factor (FCI), Fidelity Level (FL), Corrected Fidelity Level (CFL), User Reported (UR), Relative frequency of citation (RFC), Relative Importance Level (RIL), Use Value (UV)

APPLIED ECOLOGY AND ENVIRONMENTAL RESEARCH 18(6):7953-7979.

http://www.aloki.hu • ISSN 15891623 (Print) • ISSN 17850037 (Online)

DOI: http://dx.doi.org/10.15666/aeer/1806_79537979

(c) 2020, ALÖKI Kft., Budapest, Hungary 


\section{Discussion}

\section{Ethnobotanical knowledge of threatened species and therapeutic uses}

Utilization of plants by humans as medicines was almost started in the Middle of Paleolithic era some 60,000 years back (Shipley and Kindscher, 2016). Plants can also be used as herbal medicines and the quantity of their usage in daily life varies because these plants are selected randomly and screened for different type of ailments (Gaoue et al., 2017). Traditional knowledge as per practice transferred from generation to generation through verbal and oral means of communication that is why evaluation on scientific basis got prime importance (Aziz et al., 2017; Polat et al., 2017). Data collected represent different life forms as herbs, shrubs and trees indicated presence at various localities of the selected sites (Mahmood et al., 2013).

Plants were generally used as stimulant, anthelmintic, cardiovascular diseases, febrifuge, diuretics, vermifuge, sedative and even antidote as this was previously reported by Mahmood et al. (2013) with few new species noticed in the current study. Cichorium intybus L. belongs to family Asteraceae used as a stimulant (Nandagopal and Kumari, 2007). Various plant species like Convolvulus album L., Benincasa hispida L., Cucurbita pepo L., Rosa indica L., Trianthema portulacastrum L., Caralluma edulis L. and Dodonaea viscosa purpurea L. were used as anthelmintic purposes and antiparasitic properties (Amjad et al., 2017). Current studies reported plants such as Trianthema portulacastrum L., and Allium cepa L. are the best known for cardiovascular problems as it is good for the blood flow and pressure (Ugulu, 2011). Debregeasia salicifolia $\mathrm{L}$. was considered the best against febrifuge.

Number of researchers reported the importance of different plants such as Heliotropium strigosum L., Xanthium strumarium L., Trichodesma indicum L., Benincasa hispida L., Cyperus rotundus L., Crotolaria burhia L., Avena sativa L., Rosa indica L., Albizia lebbek L., Trianthema portulacastrum L., Caralluma edulis L., Grewia asiatica L., Micromeria biflora L. and Duchesnea indica L. as diuretic (Mahmood et al., 2013; Bhakshu et al., 2008; Verma and Kumar, 2011; Zia-Ul-Haq et al., 2012; Amjad et al., 2017). The plants that were used for vermifuge purposes includes Indigofera heterantha L., Portulaca quadrifida L., Pongamia pinnata L. and Cyperus rotundus L. (Ghumare et al., 2014). Xanthium strumarium L. and Datura innoxia L., were used for sedative purpose. Current studies revealed few plants as antidote such as Taraxacum officinale L., Artemisia scoparia L., Calotropis procera L., Ranunculus muricatus L., Melia azedarach L. and Trichodesma indicum L.

One of the most noticed diseases from the study area were rheumatism and many plants were used for curing this disease like Cuscuta reflexa L., Pongamia pinnata L., Amaranthus viridis L., Calotropis procera L. and Solanum surattense L. Ghumare et al. (2014) noticed the first two species and rest of all are reported in this study. For blood purification species like Dodonaea viscosa purpurea L., Scandix pectenveneris L., Grewia asiatica L., Morus nigra L., Albizia lebbek L., Melia azedarach L., Eichhornia crassipes L., Punica granatum L., Cynodon dactylon L., Crotolaria burhia L. and Heliotropium strigosum L. (Bhakshu et al., 2008).

Many diseases such as hemorrhagic septicemia, jaundice, wound healing, severe burns and skin treatments of various types were also found to be treated with different plant species like Quercus incana L. Jaundice was also treated by different plants such as Debregeasia salicifolia L., Berberis lyceum L., Solanum nigrum L., Carthamus oxyacantha L., Eclipta prostrate L., Trianthema portulacastrum L., Portulaca oleracea 
L. Citrullus colocynthis L. Indigofera linifolia L. while Mirabilis jalapa L. for skin treatment. Similarly, this study also signifies that Lonicera quinquelocularis L., Berberis lyceum L. and Malvastrum coromandelianum L. were observed to be useful in wound healing (Amjad et al., 2017).

Some plants were also reported for treating various diseases like piles, dysentery, stomach problems, vomiting, measles, diarrhea, asthma, diabetes, liver tonic, tooth aches, urinary problems and sexual diseases like Zanthoxylum armatum L., Tamarix dioica L., Abutilon indicum L., Portulaca quadrifida L., Alhagi maurorum L., and Convolvulus album L., are used for the treatment of piles (Amjad et al., 2017; Mahmood et al., 2013). For dysentery, some plants were useful such as Trichodesma indicum L., Cyperus rotundus L., Dalbergia sissoo L., Cynodon dactylon L., Punica granatum L., Calotropis procera L., Cynodon dactylon L. In current studies, Citrullus colocynthis L. and Zanthoxylum armatum L. were shown to be used for releasing stomach pain while Micromeria biflora L. and Bryophyllum pinnatum L. are used against vomiting that were present in both Gujranwala and Wazirabad. For curing measles Zizyphus nummularia L. and Asparagus racemosus L. were found efficient. Amjad et al. (2017) reported many plants present in this region were used for treatment of diarrhea which were Melilotus indica L., Vinca major L., Conyza aegyptiaca L., Tamarix dioica L., Withania somnifera L., Cassia fistula L., Mentha spicata L., Cyperus rotundus L., Trichodesma indicum L. and Bryophyllum pinnatum L. and these all are present in the study area. For asthma Euphorbia hirta, Albizia lebbek, Trianthema portulacastrum L., Solanum surattense L., Asparagus racemosus L. and Fumaria indica L. were beneficial plants found in the study area. Plants which were found associated with the treatment of diabetes were Melilotus indica L., Vinca major L., Taraxacum officinale L., Parthenium hysterophorus L., Caralluma edulis L., Syzygium cumini L. and Cuscuta reflexa L. grown at various places in Kamonki and Wazirabad. Heliotropium strigosum L., Avena sativa L., Portulaca oleracea L., Trianthema portulacastrum L., Asparagus racemosus L. and Duchesnea indica L. acts as liver tonic. For tooth aches Conyza aegyptiaca L., Salvadora oleoides L., Nerium oleander L., Justicia adhatoda L., Pongamia pinnata L. and Cuscuta reflexa L. were important which are also present in Wazirabad, Kamonki and Gujranwala. For urinary infection some plants such as Chenopodium album L. and Hibiscus rosasinensis L. were found in the study area.

Plants used as anti-inflammatory purposes included Trichodesma indicum L., Lepidium didymium L. and Jasminum officinale L., (Tareen et al., 2016). Most of the plants were linked with treatment of common diseases like fever and constipation. For fever, Convolvulus album L., Trichodesma indicum L., Dalbergia sissoo L., Bumbusa arundinacea L., Marva parviflora L., Eucalyptus globulus L., Morus nigra L., Justicia adhatoda L., Achyranthes aspera L., Artemisia scoparia L., Solanum surattense L., Conyza aegyptiaca L., Fumaria indica L., Malvastrum coromandelianum L. Debregeasia salicifolia $\mathrm{L}$ were used found at various places of selected sites and for constipation the beneficial plants found are Micromeria biflora L., Grewia asiatica L., Taraxacum officinale L., Mirabilis jalapa L., Hibiscus rosasinensis L., Zizyphus nummularia L., Rosa indica L., Alhagi maurorum L., Citrullus colocynthis L. and Convolvulus album L. Interestingly a unique species and only one was also recorded for treating epilepsy from this region that was Benincasa hispida L. that belongs to the family Cucurbitaceae.

Plants were also reported to be used for various other aspects especially for aesthetic beauty, decorative purposes and as ornamentals etc., along as food, shelter, clothing, dyes, coloring etc. Artemisia scoparia L. a member of the family Asteraceae was used 
in preventing hair loss. For this, the plant extract should be mixed with coconut oil and then boiled. It can be applied on the head to strengthen the hairs and to prevent them from becoming white. Calendula arvensis L., another member of the same family was used in weddings for decorative purposes. Melia azedarach L. and Malvastrum coromandelianum L. are the members of family Malvaceae used as ornamentals in various places of Gujranwala. Similarly, Cassia fistula L. and Acacia modesta L. members of family Mimosaceae are grown at various places in the selected sites of the Gujranwala region. Different plants have been used differently by the people such as Chenopodium is used as fodder crop for animals and it is used in the case of constipation. Cucurbeta peppo L. the favorite vegetable of the summer was found in the study area. It is the member of family Cucerbitaceae, other members of the same family were Cucumus melo L. and Benincasa hispada L. that were used as vegetables. Acacia modesta L. and Acacia nilotica L. members of family Mimosaceae were wildly found in the study area and are used in making and as additive for gums.

\section{Quantitative indices, phytochemical diversity and their role for conservation}

The relative frequency of citation (RFC) varies from 1 (means the number of useful plants responded by the respondents) to 0 (i.e. no one reported the plants uses). The lowest value for RFC calculated by the gathered data is 0.001 of plant Lonicera quinquelocularis which is used for the treatment of improving vision, removing cataract, as ophthalmic agent, wound healing purpose and the highest value is 0.78 of plant Nerium oleander used in the treatment for abortions, toothache and earache. Informants consensus factor (FCI) also describes the number of used reports for a said disease category and in this study it ranged from 10 to 40 . The lowest value of FCI was 10 for Cucumus melo which was used in the treatment of eczema, dysuria, leucorrhea and also acts as laxative. The highest value was 37 of plants Indigofera heterantha and Quercus incana used in treatment of hemorrhagic septicemia and joint pain.

Relative importance level (RIL) indicated the eminence of the species in the selected sites of study area. RIL varies from 0 (not much importance) to 1.0 (medicinal importance). The lowest value recoded by the data was 0.34 of plant Solanum surattense used for rheumatism, fever, asthma and the highest value was 0.99 of different plants like Marva parviflora, Solanum nigrum, Tamarix dioica, Lonicera quinquelocularis and Zanthoxylum armatum were being used of carminative, dyspepsia, stomach pains and piles (Umair et al., 2019). Fidelity level (FL) showed uses of specific plants to treat particular diseases. The fidelity count of plants in the study area ranges up to 90 that mark their importance in the field of medicines. Benincasa hispida have the lowest value calculated through gathered data i.e. 46.35 used for different problems laxative, diuretic, tonic, anthelminthic, epilepsy and the highest value is 98 for Marva parviflora, Justicia adhatoda and Tamarix dioica used in piles and diarrhea. Corrected fidelity level (CFL) is a correction factor that links RIL with FL and shows relative importance of different plants found in the study area. Different respondents give useful information which is an important aspect of the study. The lower value of CFL was 12 for Eichhornia crassipes, having different uses such as emollient, blood purifier and the highest value was 88 of Justicia adhatoda plant that was used as abortifacient, for cold, cough, flu and toothache.

User reports (UR) is the total number of users reported about a particular plant and this ranges between 1 and 20 mostly i.e. up to 20 users reported about a particular disease to be treated by the particular plant. The lowest numbers of users reported were 
11, for plant Trichodesma indicum, which was used as antidote, diuretic, against dysentery and fever and the highest numbers of users reported were 380, for plant Syzygium cumini which was helpful in diabetes. The significance of each species was calculated by UV (use value) especially in the case of herbal drinks (Etongo et al., 2017). Use value (UV) of the plants was also beneficial for the studying of medicinal plants and in this study it ranges from 0 to 1 i.e. little to more importance in the field of medicines. The value of UV of plant Citrullus colocynthis was 0.1 which is the lowest value used in the treatment of amenorrhea, constipation, stomach aches, jaundice, also used as purgative, and the highest value of UV was 0.98 of plant Trichodesma indicum used for dysentery, fever and diarrhea.

Secondary metabolites are vital ingredients present in plants were quantified qualitatively using standard procedures. The severity of chemicals present in the selected sites of plants indicates their importance. About Twenty different plants were analyzed for phytochemical analysis. Punica granatum, Chenopodium album, Jasminum officinale, Cyperus rotundus, Morus nigra, Bryophyllum pinnatum and many others showed presence/indication of alkaloids, flavonoids, tannins, steroids, terpenoids, saponins, phenols and resins that are the major source of efficacy of various bioactive compounds effective against number of ailments. Further, these plants rich in biochemical constituents recommended for further characterization through GCMS or HPLC to gather more information about biochemical structure and their ingredients ultimate impact against ailments which are still considered as complex to treat completely. But in some plants such as Quercus incana, Bambusa arundinacea and Syzygium cumini compounds like terpenoids, saponins and steroids did not show any indication that might be due to variation in geographical area, topology, soil physical and chemical factors, climatic factors, and/or species or genetic variation. One of the major reasons might be industrialization that causes accumulation of toxic chemicals, industrial waste and heavy metals that needs thorough examination.

\section{Conclusion}

In conclusion, the anthropocentric activities due to industry and urbanization may alter or degrade phytochemical diversity that ultimately change efficacy of particular extracts. Current studies revealed 100 different species of 52 families, where Asteraceae was dominant. Different life forms recorded i.e., 55\% herbs, $27 \%$ shrubs, $15 \%$ trees, $2 \%$ grasses and $1 \%$ weeds, $85 \%$ wild and $15 \%$ cultivated. Leaves were predominantly used as $77 \%$. Plants were found effective against fever, as laxatives, emollients, against constipation, as blood purifiers, and against cough and cold etc. Informant consensus factor for Cucumus melo showed the lowest value while Indigofera heterantha and Quercus incana represent the highest. Involvement of local community with public sector for implementing policies and guidelines according to Biodiversity action plan of Pakistan is required to be practiced to achieve environmental sustainability.

Acknowledgements. Authors are thankful to Prof. Dr. Mushahid Anwar, Dean FS/Chairperson, Department of Geography, University of Gujrat, Gujrat, Pakistan for Gujranwala map cartography and Ms. Tayyaba Khalil, Incharge Herbarium, Department of Botany, University of Gujrat, Gujrat, Pakistan for identification and herbarium specimen preparation. 


\section{REFERENCES}

[1] Ahmed, E., Arshad, M., Saboor, A., Qureshi, R., Mustafa, G., Sadiq, S. (2013): Ethnobotanical appraisal and medicinal use of plants in Patriata, New Murree, evidence from Pakistan. - Journal of Ethnobiology and Ethnomedicine 9: 1-10.

[2] Aiyegoro, O. A., Okoh, A. I. (2010): Preliminary phytochemical screening and in vitro antioxidant activities of the aqueous extract of Helichrysum longifolium DC. - BMC Complementary and Alternative Medicine 10: 1-21.

[3] Akhtar, N., Begum, S. (2009): Ethnopharmacological important plants of Jalala, district Mardan, Pakistan. - Pakistan Journal of Plant Sciences 15: 95-100.

[4] Alexiades, M. N., Sheldon, J. W. (1996): Selected Guidelines for Ethnobotanical Research: A Field Manual. - New York Botanical Garden, Bronx, NY.

[5] Ali-Shtayeh, M. S., Yaniv, Z., Mahajna, J. (2000): Ethnobotanical survey in the Palestinian area: a classification of the healing potential of medicinal plants. - Journal of Ethnopharmacology 73: 221-232.

[6] Alsaidy, H. A. M. (2013): Evaluation efficiency of garlic and colocynth as crude extraction and some antifungal effects on fungus Candida Albicans, which was isolated from patients of Diyala province towns. Iraq. - International Journal of Agricultural Science and Research 3: 45-51.

[7] Amiri, M. S., Joharchi, M. R. (2016): Ethnobotanical knowledge of Apiaceae family in Iran: A review. - Avicenna Journal of Phytomedicine 6: 1-15.

[8] Amjad, M. S., Arshad, M., Saboor, A., Page, S., Chaudhari, S. K. (2017): Ethnobotanical profiling of the medicinal flora of Kotli, Azad Jammu and Kashmir, Pakistan: Empirical reflections on multinomial log it specifications. - Asian Pacific Journal of Tropical Medicine 10: 503-514.

[9] Aziz, M. A., Khan, A. H., Adnan, M., Izatullah, I. (2017): Traditional uses of medicinal plants reported by the indigenous communities and local herbal practitioners of Bajaur Agency, Federally Administrated Tribal Areas, Pakistan. - Journal of Ethnopharmacology 198: 268-281.

[10] Bhakshu, L. M., Ratnam, K. V., Venkataraju, R. R. (2008): Medicinal properties and antimicrobial activity of Crotalaria madurensis var. Kurnoolica. - Ethnobotanical Leaflets 2: 104-109.

[11] Brandão, M. G. L., Acúrcio, F. A., Montemor, R. L. M., Marlière, L. D. P. (2006): Complementary/alternative medicine in Latin America: use of herbal remedies among a Brazilian metropolitan area population. - Journal of Complementary and Integrative Medicine 3: 1535-3840.

[12] Etongo, D., Djenontin, I. N. S., Kanninen, M., Glover, E. K. (2017): Assessing use values and relative importance of trees for livelihood values and their potentials for environmental protection in Southern Burkina Faso. - Environment, Development and Sustainability 19: 1141-1166.

[13] Fahey, J. W. (2005): Moringa oleifera: a review of the medical evidence for its nutritional, therapeutic, and prophylactic properties. Part 1. - Trees for Life Journal 1: 115.

[14] Farhan, H., Rammal, H., Hijazi, A., Badran, B. (2012): Preliminary phytochemical screening and extraction of polyphenol from stems and leaves of a Lebanese plant Malva parviflora L. - International Journal of Current Pharmaceutical Research 4: 55-59.

[15] Friedman, J., Yaniv, Z., Dafni, A., Palewitch, D. (1986): A preliminary classification of the healing potential of medicinal plants, based on a rational analysis of an ethnopharmacological field survey among Bedouins in the Negev Desert, Israel. - Journal of Ethnopharmacology 16: 275-287.

[16] Gaoue, O. G., Coe, M. A., Bond, M., Hart, G., Seyler, B. C., McMillen, H. (2017): Theories and major hypotheses in ethnobotany. - Economic Botany 71: 269-287. 
[17] Ghumare, P., Jirekar, D. B., Farooqui, M., Naikwade, S. D. (2014): A review of Pongamia pinnata - an important medicinal plant. - Current Research in Pharmaceutical Sciences 4: 44-47.

[18] Gulshan, A. B., Dasti, A. A., Hussain, S., Atta, M. I., Amin-ud-Din, M. (2012): Indigenous uses of medicinal plants in rural areas of Dera Ghazi Khan, Punjab, Pakistan. - ARPN Journal of Agricultural and Biological Science 7: 750-762.

[19] Gupta, A., Gautam, M. K., Singh, R. K., Kumar, M. V., Rao, C. V., Goel, R. K. (2010): Immunomodulatory effect of Moringa oleifera Lam. extract on cyclophosphamide induced toxicity in mice. - Indian Journal of Experimental Biology 48: 1157-1160.

[20] Heinrich, M., Edwards, S., Moerman, D. E., Leonti, M. (2009): Ethnopharmacological field studies: a critical assessment of their conceptual basis and methods. - Journal of Ethnopharmacology 124: 1-17.

[21] Jaffer, H. J., Mahaed, M. J., Jawad, A. M., Naji, A., Al-Naib, A. (1983): Phytochemical and biological screening of some Iraqi plant. - Fitoterapialix (in Arabic).

[22] Khan, M., Hussain, F. (2013): Conservation status of plant species in Township Takht-eNasrati, District Karak, Khyber Pakhtun Khawa, Pakistan. - International Journal of Biodiversity and Conservation 5: 20-26.

[23] Khan, W., Ahmad, H., Haq, F., Islam, M., Bibi, F. (2012): Present status of moist temperate vegetation of Thandiani forests district Abbottabad Pakistan. - International Journal of Biosciences 2: 80-88.

[24] Mahmood, A., Mahmood, A., Malik, R. N., Shinwari, Z. K. (2013): Indigenous knowledge of medicinal plants from Gujranwala district, Pakistan. - Journal of Ethnopharmacology 148: 714-723.

[25] Nandagopal, S., Kumari, B. R. (2007): Phytochemical and antibacterial studies of chicory (Cichorium intybus L.) - a multipurpose medicinal plant. - Advances in Biological Research 1: 17-21.

[26] Polat, R., Cakilcioglu, U., Selvi, S., Türkmen, Z., Kandemir, A. (2017): The anatomical and micromorphological properties of three endemic and medicinal Salvia species (Lamiaceae) in Erzincan (Turkey). - Plant Biosystems - An International Journal Dealing with all Aspects of Plant Biology 151: 63-73.

[27] Samie, A., Obi, C. L., Bessong, P. O., Namrita, L. (2005): Activity profiles of fourteen selected medicinal plants from Rural Venda communities in South Africa against fifteen clinical bacterial species. - African Journal of Biotechnology 4: 1443-1451.

[28] Shanley, P., Luz, L. (2003): The impacts of forest degradation on medicinal plant use and implications for health care in eastern Amazonia. - BioScience 53: 573-584.

[29] Shihata, I. M. (1951): A pharmacological study of Anagallis Arvensis. - MD Vet Doctoral Dissertation Thesis, Cairo University.

[30] Shipley, G. P., Kindscher, K. (2016): Evidence for the paleoethnobotany of the Neanderthal: a review of the literature. - Scientifica 29: 1-12.

[31] Sofowora, A. (1993): Screening Plants for Bioactive Agents. Medicinal Plants and Traditional Medicine in Africa. 2nd Ed. - Spectrum Books Ltd, Sunshine House, Ibadan, pp. 134-156.

[32] Tardío, J., Pardo-de-Santayana, M. (2008): Cultural importance indices: a comparative analysis based on the useful wild plants of Southern Cantabria (Northern Spain). Economic Botany 62: 24-39.

[33] Tareen, N. M., Saeed-ur-Rehman, M. A., Shinwari, Z. K., Bibi, T. (2016): Ethnomedicinal utilization of wild edible vegetables in district Harnai of Balochistan province, Pakistan. - Pakistan Journal of Botany 48: 1159-1171.

[34] Ugulu, I. (2011): Traditional ethnobotanical knowledge about medicinal plants used for external therapies in Alasehir, Turkey. - International Journal of Medicinal and Aromatic Plants 1: 101-106. 
[35] Umair, M., Altaf, M., Bussmann, R. W., Abbasi, A. M. (2019): Ethnomedicinal uses of the local flora in Chenab riverine area, Punjab province Pakistan. - Journal of Ethnobiology and Ethnomedicine 15: 1746-4269.

[36] Verma, S. K., Kumar, A. (2011): Therapeutic uses of Withania somnifera (ashwagandha) with a note on withanolides and its pharmacological actions. - Asian Journal of Pharmaceutical and Clinical Research 4: 1-4.

[37] Wazir, S. M., Saima, S., Dasti, A. A., Subhan, M. (2007): Ethnobotanical importance of salt range species of district Karak, Pakistan. - Pakistan Journal of Plant Sciences 13: 2931.

[38] Zaman, S., Hazrat, A., Ullah, S. (2013): Ethnobotanical survey of medicinal plants from Township Dargai, district Malakand, Pakistan. - FUUAST Journal of Biology 3: 109113.

[39] Zereen, A., Sardar, A. A. (2013): Ethnobotanical studies of wild herbs of central Punjab, Pakistan. - Bangladesh Journal of Plant Taxonomy 20: 67-76.

[40] Zia-Ul-Haq, M., Shahid, S. A., Muhammed, S., Qayum, M., Khan, I., Ahmad, S. (2012): Antimalarial, antiemetic and antidiabetic potential of Grewia asiatica L. leaves. - Journal of Medicinal Plant Research 6: 3087-92. 